\title{
Electronic Structure of Paramagnetic $\mathrm{V}_{2} \mathrm{O}_{3}$ : Strongly Correlated Metallic and Mott Insulating Phase
}

\author{
G. Keller ${ }^{1}$, K. Held, ${ }^{2}$ V. Eyert ${ }^{1}$, D. Vollhardt, ${ }^{1}$ and V. Anisimov ${ }^{3}$ \\ ${ }^{1}$ Institut für Physik, Universität Augsburg, 86135 Augsburg, Germany \\ ${ }^{2}$ Max-Planck-Institut für Festkörperforschung, 70569 Stuttgart, Germany \\ ${ }^{3}$ Institute of Metal Physics, Ekaterinburg GSP-170, Russia
}

(Dated: Version 1, October 31, 2018)

\begin{abstract}
LDA+DMFT, the computation scheme merging the local density approximation and the dynamical mean-field theory, is employed to calculate spectra both below and above the Fermi energy and spin and orbital occupations in the correlated paramagnetic metallic and Mott insulating phase of $\mathrm{V}_{2} \mathrm{O}_{3}$. The selfconsistent DMFT equations are solved by quantum Monte Carlo simulations. Room temperature calculations provide direct comparison with experiment. They show a significant increase of the quasiparticle height in comparison with the results at $1160 \mathrm{~K}$. We also obtain new insights into the nature of the MottHubbard transition in $\mathrm{V}_{2} \mathrm{O}_{3}$. Namely, it is found to be strikingly different from that in the one-band Hubbard model due to the orbital degrees of freedom. Furthermore we resolve the puzzle of the unexpectedly small Mott gap in $\mathrm{Cr}$-doped $\mathrm{V}_{2} \mathrm{O}_{3}$.
\end{abstract}

\section{INTRODUCTION}

The phase transition between a paramagnetic metal and a paramagnetic insulator caused by the Coulomb interaction between the electrons is referred to as MottHubbard metal-insulator transition. ${ }^{1,2}$ Reliable microscopic investigations of this many-body phenomenon are known to be exceedingly difficult. Indeed, the question concerning the nature of this transition poses one of the fundamental theoretical problems in condensed matter physics. Correlation-induced metal-insulator transitions (MIT) of this type are found, for example, in transition metal oxides with partially filled bands near the Fermi level. In these systems band theory typically predicts metallic behavior. The most famous example is $\mathrm{V}_{2} \mathrm{O}_{3}$ doped with $\mathrm{Cr} ;{ }^{3-5}$ see Fig. 1. While at low temperatures $\mathrm{V}_{2} \mathrm{O}_{3}$ is an antiferromagnetic insulator (AFI) with monoclinic crystal symmetry, the high-temperature paramagnetic phase has a corundum structure. All transitions shown in the phase diagram are of first order. In the case of the transitions from the high-temperature, paramagnetic phases into the low-temperature antiferromagnetic phase this is naturally explained by the fact that the transition is accompanied by a change in crystal symmetry. By contrast, the MIT in the paramagnetic phase is iso-structural; only the ratio of the $c / a$ axes changes discontinuously. This may be taken as an indication for a predominantly electronic origin of this transition.

To explain an MIT induced by electronic correlations one can either investigate a simplified electronic manybody model to understand, at least, some of the basic features of the MIT, or employ material-specific approaches such as the density functional theory in the local density approximation (LDA). Concerning the former approach, ${ }^{1,3}$ the spin $S=1 / 2$, half-filled, single-band Hubbard $\operatorname{model}^{6-8}$ is certainly the simplest possible model

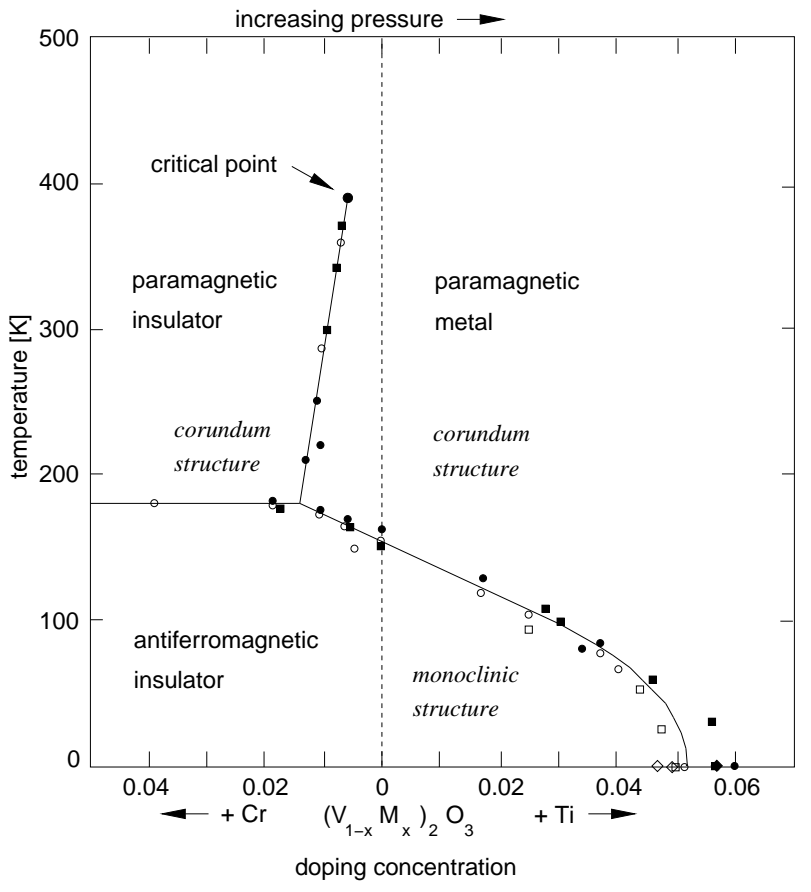

FIG. 1: Phase diagram of $\mathrm{V}_{2} \mathrm{O}_{3}$ showing the MIT as a function of pressure and of doping with $\mathrm{Cr}$ and $\mathrm{Ti}$; data points from McWhan et al. ${ }^{5}$

to be investigated. In particular, the existence of an MIT in the paramagnetic phase of the half-filled Hubbard model had been investigated already in the early work of Hubbard. ${ }^{6,9}$ However, while the Hubbard I and III approximations $^{6,9}$ describe the insulating phase rather well, they do not describe a Fermi liquid phase on the metallic side. On the other hand, the Gutzwiller approximation provides a picture of the break-down of the Fermi 
liquid phase as indicated by the collapse of the quasiparticle peak and the simultaneous divergence of the effective mass at a critical value of the Coulomb interaction $U_{c}$ (Brinkman-Rice transition). ${ }^{10}$ However, within this framework one cannot describe the Hubbard bands which are essential both in the strongly correlated metallic phase below $U_{c}$ and in the insulating phase above $U_{c}$. With these limitations, the details of the MIT in the Hubbard model remained unclear, except for the onedimensional case ${ }^{11}$ which is very particular since it always describes an insulator, i.e., $U_{c}=0^{+}$.

During the last few years, our understanding of the MIT in the one-band Hubbard model has considerably improved due to the development of dynamical meanfield theory (DMFT) ${ }^{12,13}$ Within DMFT the electronic lattice problem is mapped onto a self-consistent singleimpurity Anderson model. ${ }^{13}$ This mapping becomes exact in the limit of infinite coordination number ${ }^{12}$ and allows one to investigate the dynamics of correlated lattice electrons non-perturbatively at all interaction strengths. This is of essential importance for a problem like the MIT which occurs at a Coulomb interaction comparable to the electronic band-width. In particular, DMFT provides a framework for deriving a coherent picture of the electronic spectrum at all energy scales, i.e., of the incoherent features at high energies (Hubbard bands), ${ }^{9}$ and the coherent quasiparticle behavior at low energies. ${ }^{7,14}$ At $T=0$, the transition from the metallic to the insulating state is signaled by a divergence of the effective mass and the collapse of the Fermi liquid quasiparticle peak at the Fermi energy for Coulomb interaction $U \rightarrow U_{c}^{-} \cdot{ }^{13,15-17}$ DMFT furthermore revealed the coexistence of the metallic and the insulating phase below a critical point at temperature $T_{c}$, such that there is a first order phase transition in agreement with the experimental observation for $\mathrm{V}_{2} \mathrm{O}_{3}$. To investigate the MIT in $\mathrm{V}_{2} \mathrm{O}_{3}$, Rozenberg et al. ${ }^{18}$ applied DMFT to the one-band Hubbard model. The influence of orbital degeneracy was studied by means of the two- ${ }^{19-21}$ and three-band ${ }^{20}$ Hubbard model for the semicircular density of states (DOS) of a Bethe lattice. Most recently, a detailed analysis ${ }^{22}$ of the conductivity change demonstrated that, except for a very narrow region directly at the critical point, the critical exponents are of the liquid-gas transition type, in accordance with a Landau theory for the Mott transition within DMFT. ${ }^{15,23,24}$

Although the Hubbard model is able to explain certain basic features of the Mott-Hubbard MIT in $\mathrm{V}_{2} \mathrm{O}_{3}$ and its phase diagram, it cannot explain the physics of that material in any detail. Clearly, a realistic theory of $\mathrm{V}_{2} \mathrm{O}_{3}$ must take into account the complicated electronic structure of this system. In our previous work, ${ }^{25}$ we therefore applied the LDA+DMFT scheme to study the MIT in paramagnetic $\mathrm{V}_{2} \mathrm{O}_{3} \cdot{ }^{26,27}$ With LDA spectra calculated for the crystal structure of metallic $\mathrm{V}_{2} \mathrm{O}_{3}$ and insulating $\left(\mathrm{V}_{0.962} \mathrm{Cr}_{0.038}\right)_{2} \mathrm{O}_{3}$ as input for the subsequent three-band DMFT(QMC) calculations, we found an MIT, or rather a sharp crossover, at $U \approx 5 \mathrm{eV}$.
Due to restrictions in computer resources, the QMC calculations in Ref. 25 were done at $T=1160 \mathrm{~K}$. Subsequently, extensive QMC simulations at temperatures down to $T \approx 300 \mathrm{~K}$ were performed to make possible a comparison between experiment and theory at experimentally relevant temperatures. Those computations yielded spectra with a quasiparticle peak at the Fermi edge considerably stronger than that at $1160 \mathrm{~K}$ and were in contrast to the existing photoemission measurements. This puzzle was finally resolved by recent improvements in photoemission spectroscopy (PES) experiments which allowed one to perform high-energy, bulk sensitive PES, displaying a prominent peak at $E_{F}$ in essential agreement with the LDA+DMFT results. ${ }^{28}$

In this paper, we provide details of our calclations reported in Ref. 28, present LDA+DMFT spectra for $300 \mathrm{~K}, 700 \mathrm{~K}$ and $1160 \mathrm{~K}$ both below and above the Fermi edge, and compare them to PES and XAS measurements. Based on calculations of spin and orbital occupations, we then discuss the properties of the ground state. In particular, the nature of the MIT turns out to be rather distinct from that in a one-band model, i.e., we find that the effective mass in the $a_{1 g}$ orbitals does not diverge at the MIT transition.

The paper is organized as follows: In Section II the LDA band structure, based on the experimental corundum crystal structure, and the relevance of $\mathrm{V}-\mathrm{V}$ pairs are discussed. Electronic correlations are taken into account by DMFT as described in Section III. The resulting LDA+DMFT spectra are presented in Section IV, including a discussion of the dependence on temperature and the Hund's rule exchange coupling. The pecularities of the MIT in $\mathrm{V}_{2} \mathrm{O}_{3}$ and the differences to the MIT of the one-band Hubbard model are worked out in Section V. A detailed comparison with the experimental spectra follows in Section VI. A summary and outlook is finally provided in Section VII.

\section{CRYSTAL AND ELECTRONIC STRUCTURE}

In the paramagnetic metallic phase stoichiometric $\mathrm{V}_{2} \mathrm{O}_{3}$ crystallizes in the corundum structure, which has a trigonal lattice and space group $R \overline{3} c\left(D_{3 d}^{6}\right)$ with lattice constants $a=4.9515 \AA$ and $c=14.003 \AA .^{29}$ Vanadium and oxygen atoms occupy the Wyckoff positions (12c) and (18e) with internal parameters $z_{V}=0.34630$ and $x_{O}=0.31164$, respectively, which deviate markedly from the value $1 / 3$ assumed in an ideal hexagonal arrangement. ${ }^{29}$ Within the corundum structure the vanadium atoms are arranged in pairs along the hexagonal $c$-axis, which can be derived from an ideal chain structure by introducing vacancies at every third site. ${ }^{30}$ The oxygen atoms form distorted octahedra around the vanadium sites. While the V-V pair along the hexagonal caxis shares octahedral faces, the octahedra are interlinked via edges and corners perpendicular to this axis, ${ }^{29,30}$ see Fig. 2. 


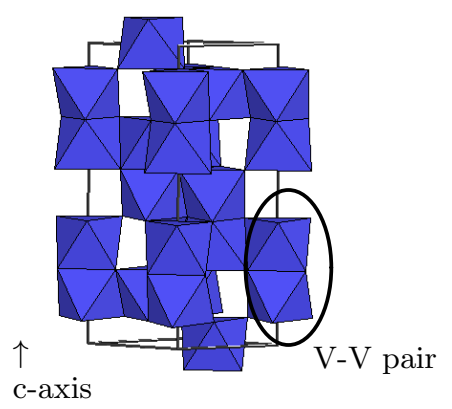

FIG. 2: Crystal structure of $\mathrm{V}_{2} \mathrm{O}_{3}$.

In the Cr-doped paramagnetic insulating phase the lattice symmetry is preserved, but the crystal structure parameters change slightly. In particular, for $\left(\mathrm{V}_{0.962} \mathrm{Cr}_{0.038}\right)_{2} \mathrm{O}_{3}$ the lattice constants amount to $a=$ $4.9985 \AA$ and $c=13.912 \AA$ and the positional parameters are $z_{V}=0.34870$ and $x_{O}=0.30745$, respectively. ${ }^{29}$ All these changes combine into a distinct displacement pattern: As compared to the metallic phase the shared octahedral faces between the $\mathrm{V}-\mathrm{V}$ pair shrink, while those octahedral faces pointing to the opposite side along the $c$-axis, i.e., towards the aforementioned vacancies, are enlarged. At the same time, the vanadium atoms shift away from the shared faces of the V-V pair such that the distances within the V-V pair increase upon Cr-doping, even though the $c$-axis lattice constant decreases. The increased $a$-axis lattice constant directly leads to enhanced vanadium distances within the $a b$-plane. As a net result, all nearest-neighbor vanadium distances are enlarged for insulating $\left(\mathrm{V}_{0.962} \mathrm{Cr}_{0.038}\right)_{2} \mathrm{O}_{3}$. Hence, we expect a reduction of the bandwidth especially of the $t_{2 g}$-derived bands. From a comparison of pure and doped $\mathrm{V}_{2} \mathrm{O}_{3}$ as well as $\mathrm{Cr}_{2} \mathrm{O}_{3}$, Dernier ${ }^{29}$ concluded that the metallic properties are intimately connected with the vanadium hopping within the $a b$-plane rather than with hopping processes between the $\mathrm{V}-\mathrm{V}$ pairs along the $z$-axis.

In a first step LDA band structure calculations ${ }^{31,32}$ were performed, which used the augmented spherical wave (ASW) method. ${ }^{33,34}$ Figs. 3,5,6, and 7 show these bandstructures along selected high symmetry lines (Fig. 4) within the first Brillouin zone of the hexagonal lattice and the densities of state (DOS) for $\mathrm{V}_{2} \mathrm{O}_{3}$ and $\left(\mathrm{V}_{0.962} \mathrm{Cr}_{0.038}\right)_{2} \mathrm{O}_{3}$, respectively.

In total our results are in good agreement with those published by Mattheiss. ${ }^{30}$ In particular, while the $\mathrm{O} 2 p$ derived bands show up in the range between -9 and -4 $\mathrm{eV}$, the $\mathrm{V} 3 d$ dominated states fall, due to the octahedral surrounding with oxygen, into two groups of bands: $t_{2 g}$ and $e_{g}^{\sigma}$. With this separation, the $e_{g}^{\sigma}$ bands will be empty and the $t_{2 g}$ bands partially filled with two electrons per $\mathrm{V}$ ion.

Due to the lower trigonal lattice symmetry the $t_{2 g}$ states are further split into doubly and singly degenerate $e_{g}^{\pi}$ and $a_{1 g}$ states, see Figs. 5, 7 and the scheme Fig. 8. The value of this splitting $(\approx 0.3 \mathrm{eV}$ for the

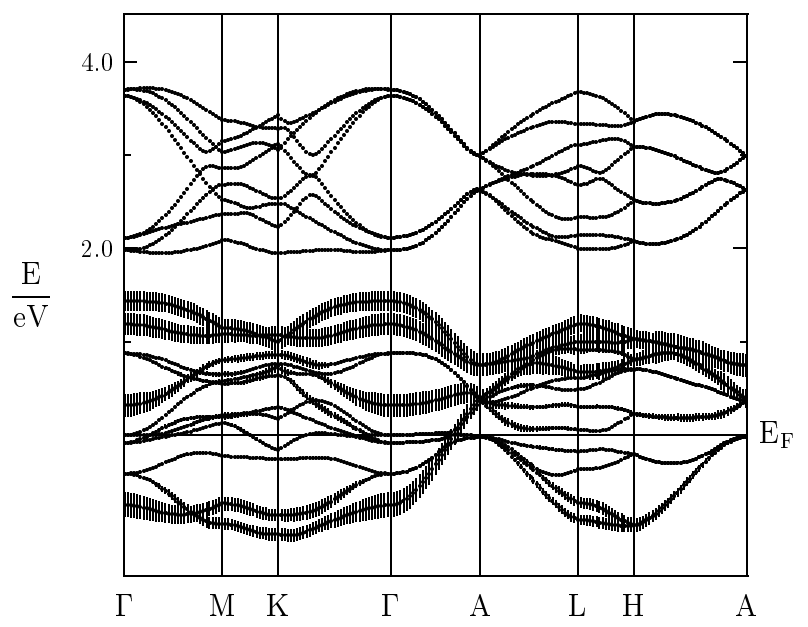

FIG. 3: Electronic bands of $\mathrm{V}_{2} \mathrm{O}_{3}$ along selected symmetry lines within the first Brillouin zone of the hexagonal lattice, Fig. 4. The width of the bars given for each band indicates the contribution from the $a_{1 g}$ orbitals.

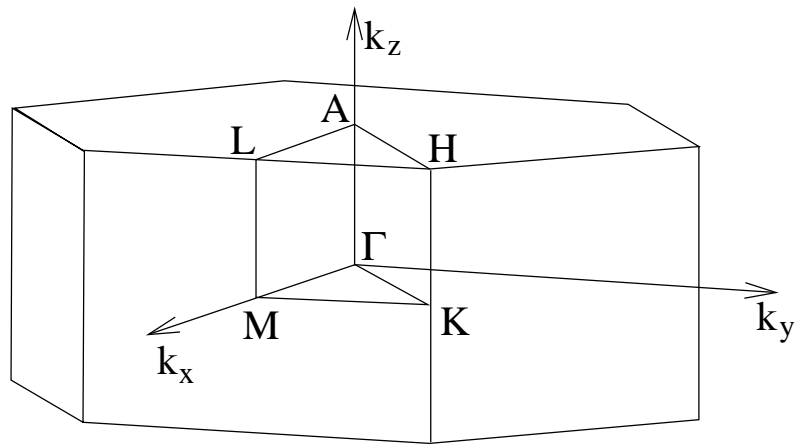

FIG. 4: First Brillouin zone of the hexagonal lattice.

centers of gravity) is much smaller than the $t_{2 g}$ bandwidth $(\approx 2 \mathrm{eV})$. However, as the value of the Coulomb interaction parameter $U(U>4 \mathrm{eV})$ is larger than the bandwidth, this small trigonal splitting strongly determines the orbital ground state of the $\mathrm{V}$-ion obtained from LDA+DMFT calculations, as will be shown below. To highlight the difference between $e_{g}^{\pi}$ and $a_{1 g}$ states, we append to each band at each $\mathbf{k}$ point a bar in Fig. 3, whose length is a measure for the contribution from the $a_{1 g}$ orbitals to the respective wave function.

The changes on going to $\left(\mathrm{V}_{0.962} \mathrm{Cr}_{0.038}\right)_{2} \mathrm{O}_{3}$ are stated easily: In Figs. 6 and 7, we observe a narrowing of the $t_{2 g}$ and $e_{g}^{\sigma}$ bands of $\approx 0.2$ and $0.1 \mathrm{eV}$, respectively, as well as a slight downshift of the centers of gravity of the $e_{g}^{\pi}$ bands. However, the insulating band gap expected for a calculation with the insulating crystal structure is missing.

As already mentioned a peculiarity of the corundum crystal structure are the $c$-axis $\mathrm{V}-\mathrm{V}$ pairs. Long ago Allen $^{35}$ emphasized the importance of the intra-pair interactions for interpreting spectroscopic properties of 


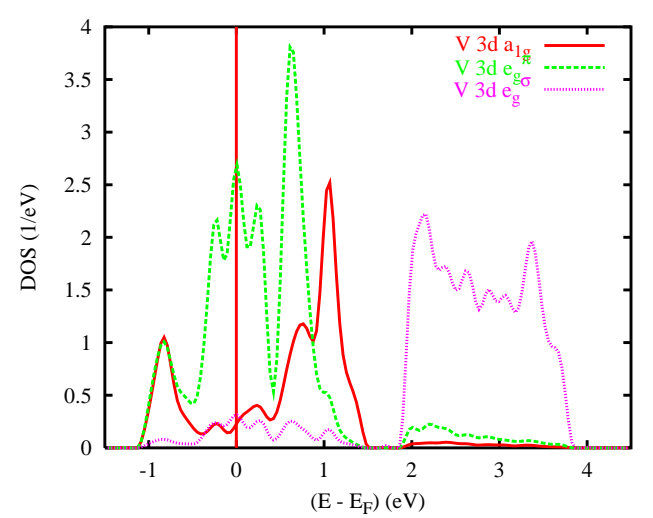

FIG. 5: Total and partial densities of states (DOS) of $\mathrm{V}_{2} \mathrm{O}_{3}$ per unit cell.

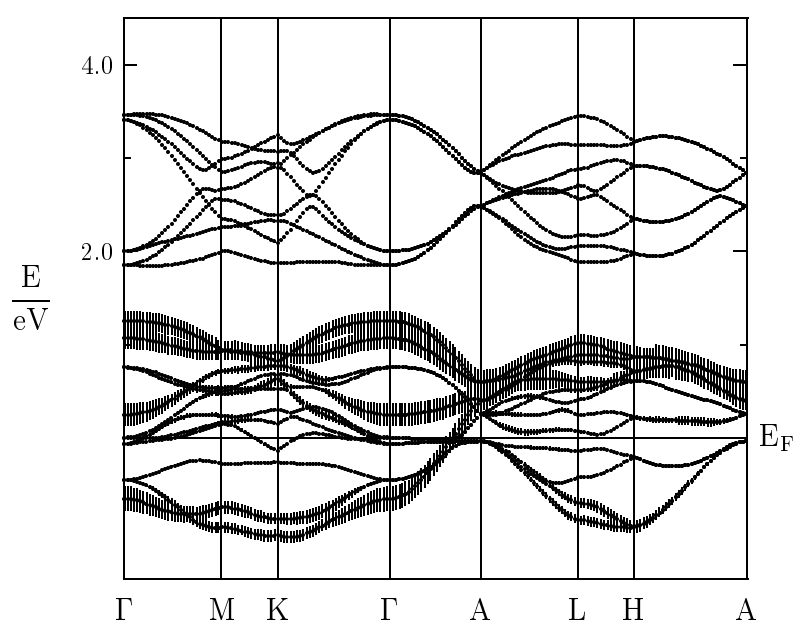

FIG. 6: Electronic bands of $\left(\mathrm{V}_{0.962} \mathrm{Cr}_{0.038}\right)_{2} \mathrm{O}_{3}$.

$\mathrm{V}_{2} \mathrm{O}_{3}$ and its solid solution with $\mathrm{Cr}_{2} \mathrm{O}_{3}$. Since the $a_{1 g}$ orbitals are directed along the c-axis, these orbitals are the ones which mediate a strong hybridization between $\mathrm{V}-\mathrm{V}$ pairs. This hybridization for the $\mathrm{V}-\mathrm{V}$ pair led Castellani et $a l .{ }^{36}$ to a model (see Fig. 8) where two of the four electrons per $\mathrm{V}-\mathrm{V}$ pair occupy a bonding molecular orbital formed by $a_{1 g}$ orbitals, leaving two electrons (one per site) in a partially filled twofold-degenerate $e_{g}^{\pi}$ band. That results in a spin- $\frac{1}{2}$ orbitally degenerate state per $\mathrm{V}$ ion with complicated orbital and spin ordering pattern explaining the unusual properties of the low-temperature antiferromagnetic phase.

Indeed, Figs. 3 and 5 show some splitting of the $a_{1 g}$ bands, in particular, between the $\mathrm{M}$ and the $\mathrm{K}$ point of the bandstructure, as one would expect from the formation of a chemical bond. But, the situation is far more complicated than a simple chemical bonding of the $a_{1 g}$ band into a bonding and an anti-bonding band: There is some additional spectral weight near $E_{F}$ (e.g., in the vicinity of the $\Gamma$ point). Moreover, there is not even a low-lying "bonding" $a_{1 g}$ band in parts of the Brillouin

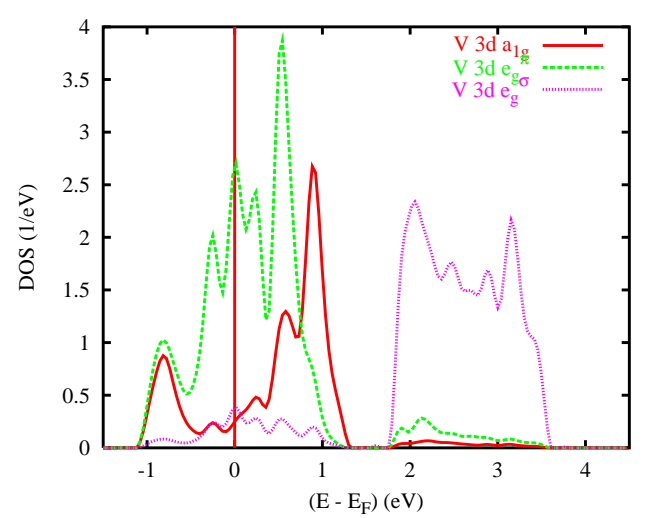

FIG. 7: Total and partial densities of states (DOS) of $\left(\mathrm{V}_{0.962} \mathrm{Cr}_{0.038}\right)_{2} \mathrm{O}_{3}$ per unit cell.

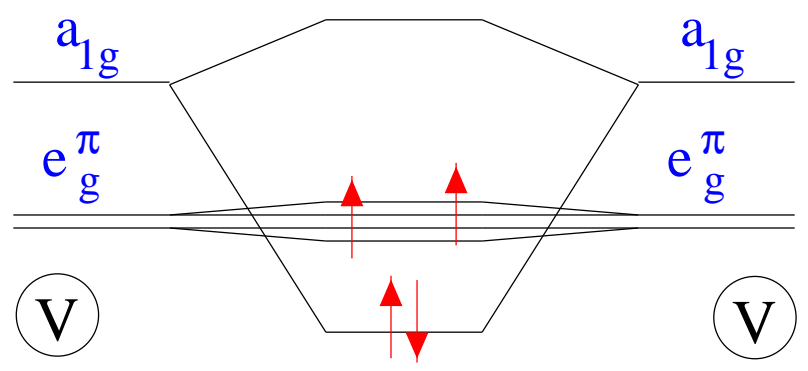

FIG. 8: Left and right: splitting of the $t_{1 g}$ orbitals in the corundum crystal structure. Middle: Formation of a chemical bond for a single $\mathrm{V}-\mathrm{V}$ pair along the $c$-axis.

zone (e.g. between the $\mathrm{H}$ and $\mathrm{A}$ point).

The Castellani et al. model ${ }^{36}$ was challenged by Park et $a .^{37}$ Based on the polarization dependence of x-ray absorption experiments they came to the conclusion that the $\mathrm{V}^{3+}$ ion is in a spin- 1 state. They also demonstrated that the orbital ground state of the ion is predominantly $e_{g}^{\pi} e_{g}^{\pi}$ with a small admixture of $e_{g}^{\pi} a_{1 g}$ configurations. This was later supported by LDA $+U$ calculations of Ezhov et al. ${ }^{38}$ where a spin-1 ground state with a $e_{g}^{\pi} e_{g}^{\pi}$ orbital configuration was obtained.

Nevertheless, the picture where the strongest hybridization parameter in $\mathrm{V}_{2} \mathrm{O}_{3}$ is the $a_{1 g}-a_{1 g}$ hopping within the $\mathrm{V}-\mathrm{V}$ pair, with all other hybridizations being much smaller, is still popular. Many theoretical studies of this material start with a (as good as possible) solution for the $\mathrm{V}-\mathrm{V}$ pair and consider the inter-pair interactions as a perturbation. ${ }^{39-41}$ All these model calculations were based on the values of hopping parameters obtained by least-square fit of LDA bands to a model Hamiltonian with nearest-neighbor hopping. Recently this problem was reexamined by Elfimov et al. ${ }^{42}$ who found that the value of the $a_{1 g}-a_{1 g}$ hopping in the $\mathrm{V}-\mathrm{V}$ pair is significantly reduced if next-nearest neighbor hoppings (which were found to be significant) are taken into account in the fit to a model Hamiltonian. Hence, one cannot consider inter-pair hoppings as a mere perturbation as it was 
taken for granted for a long time.

\section{INCLUDING ELECTRONIC CORRELATIONS VIA DMFT}

The LDA band structure of the previous section clearly fails to describe $\left(\mathrm{V}_{1-x} \mathrm{Cr}_{x}\right)_{2} \mathrm{O}_{3}$. In particular, the chromium doped compound $\left(\left(\mathrm{V}_{0.962} \mathrm{Cr}_{0.038}\right)_{2} \mathrm{O}_{3}\right.$ is an insulator whereas LDA predicts metallic behavior. The reason for this failure is that LDA deals with electronic correlations only very rudimentarily, namely, the dependence of the LDA exchange-correlation energy on the electron density is given by perturbative or quantum Monte-Carlo calculations for jellium, ${ }^{43,44}$ which is a weakly correlated system. To overcome this shortcoming, we supplement the LDA band structure by the the most important Coulomb interaction terms, i.e., the local Coulomb repulsion $U$ and the local Hund's rule exchange $J$. The local Coulomb repulsion $U$ gives rise to a genuine effect of electronic correlations, the Mott-Hubbard metal insulator transition. ${ }^{13,15-17,23,24,45}$ If the LDA bandwidth is considerably larger than the local Coulomb interaction, the LDA results are slightly modified but the system remains a metal. If the LDA bandwidth is much smaller than the local Coulomb interaction one has essentially the atomic problem where it costs an energy of about $U$ to add an electron and the system is an insulator. In between, the Mott-Hubbard metal insulator transition occurs with $\mathrm{V}_{2} \mathrm{O}_{3}$ being on the metallic side whereas $\left(\mathrm{V}_{0.962} \mathrm{Cr}_{0.038}\right)_{2} \mathrm{O}_{3}$, which has a 0.1-0.2 eV smaller bandwidth, is on the insulating side.

Interpreting the LDA band structure as a one-particle Hamiltonian $\hat{H}_{\mathrm{LDA}}^{0}$ and supplementing it with the local Coulomb interactions gives rise to the multi-band manybody Hamiltonian ${ }^{46}$

$\hat{H}=\hat{H}_{\mathrm{LDA}}^{0}+U \sum_{i m} \hat{n}_{i m \uparrow} \hat{n}_{i m \downarrow}+\sum_{i m \neq \tilde{m} \sigma \tilde{\sigma}}\left(V-\delta_{\sigma \tilde{\sigma}} J\right) \hat{n}_{i m \sigma} \hat{n}_{i n}\left(f_{z}\right)$

Here, $i$ denotes the lattice site and $\hat{n}_{i m \sigma}$ is the operator for the occupation of the $t_{2 g}$ orbital $m$ with spin $\sigma \in\{\uparrow, \downarrow\}$. The interaction parameters are related by $V=U-2 J$ which is a consequence of orbital rotational symmetry. This holds exactly for degenerate orbitals and is a good approximation in our case where the $t_{2 g}$ bands have similar centers of gravity and bandwidths. As in the local spin density approximation (LSDA), the spinflip term of the exchange interaction is not taken into account in Eq. (1). The consequences of this approximation for states in the vicinity of the Fermi energy do not seem to be large as comparative calculations using the non-crossing approximation within DMFT show. ${ }^{47}$ Furthermore, a pair hopping term proportional to $J$ is neglected since it requires that one orbital is entirely empty while another is entirely full which is a rare situation in the solid state and corresponds to highly excited states. For the Hund's rule coupling $J$ we take the constrained
LDA value $J=0.93 \mathrm{eV} \cdot{ }^{48}$ Unfortunately, such an $a b$ initio calculation is not feasible for the Coulomb repulsion $U$ since $U$ depends sensitively on screening which leads to uncertainties of about $0.5 \mathrm{eV} .{ }^{49}$ For our present purposes this uncertainty is too large since $\mathrm{V}_{2} \mathrm{O}_{3}$ is on the verge of a Mott-Hubbard metal-insulator transition, and, thus, small changes of $U$ have drastic effects. In particular, due to the small differences in the LDA band structure it is unlikely that for a $U$ value calculated by constrained LDA, $\mathrm{V}_{2} \mathrm{O}_{3}$ is metallic whereas $\left(\mathrm{V}_{0.962} \mathrm{Cr}_{0.038}\right)_{2} \mathrm{O}_{3}$ is insulating. Therefore, we adjust $U$ in such a way as to make sure that these two systems are metallic and insulating, respectively. A posteriori, we will compare the adjusted value with those calculated by constraint LDA calculations and those extracted from the experiment.

So far, we did not specify $\hat{H}_{\mathrm{LDA}}^{0}$. In principle, it should contain the valence orbitals, i.e., the oxygen $2 p$ orbitals and the five vanadium $3 d$ orbitals per atom and, maybe, some additional $s$ orbitals. However, for $\mathrm{V}_{2} \mathrm{O}_{3}$ we are in the fortunate situation that the three $t_{2 g}$ bands at the Fermi energy are well separated from the other orbitals, see Fig. 7. Therefore, it is possible to restrict ourselves to the three $t_{2 g}$ bands at the Fermi energy which are made up of the corresponding atomic vanadium $3 d$ orbitals with some admixtures of oxygen $p$ orbitals. In the case of three degenerate $t_{2 g}$ orbitals, which is close to our situation where bandwidths and centers of gravity of the $a_{1 g}$ and the doubly-degenerate $e_{g}^{\pi}$ band are very similar, the $\mathbf{k}$-integrated Dyson equation simplifies to become an integral over the $\operatorname{DOS}^{27}$

$$
G_{m}(\omega)=\int \mathrm{d} \epsilon \frac{N_{m}^{0}(\epsilon)}{\omega+\mu-\Sigma_{m}(\omega)-\epsilon} .
$$

Here $G_{m}(\omega), \Sigma_{m}(\omega)$, and $N_{m}^{0}(\epsilon)^{50}$ are the Green function, self energy, and LDA density of states, respectively, for the $t_{2 g}$ orbital $m$. In principle, $N_{m}^{0}(\epsilon)$ should contain a double counting correction, which takes into account that part of the local Coulomb interaction already included in the LDA. However, this correction results in the same effect for all three orbitals and, hence, only translates into a simple shift of the chemical potential $\mu$. This makes the issue of how to calculate the double counting correction irrelevant for the present purposes. The (shifted) $\mu$ has to be controlled according to the vanadium valency, i.e., in such a way that there are two electrons in the three bands at the Fermi energy.

Within DMFT the k-integrated Dyson equation (2) has to be solved self-consistently together with a one-site (mean field) problem which is equivalent to an Anderson impurity model with hybridization $\Delta_{m}\left(\omega^{\prime}\right)$ fulfilling ${ }^{13}$

$$
\left[G_{m}(\omega)\right]^{-1}+\Sigma_{m}(\omega)=\omega+\mu-\int_{-\infty}^{\infty} \mathrm{d} \omega^{\prime} \frac{\Delta_{m}\left(\omega^{\prime}\right)}{\omega-\omega^{\prime}} .
$$

The self-consistent solution of the Anderson impurity model given by (3) together with the Dyson equation (2) allows for a realistic investigation of materials with strongly correlated electrons. At small values of $U$ this procedure typically yields a spectrum 
with a central quasiparticle resonance at the Fermi energy and two incoherent Hubbard side bands, while at larger values of $U$ the quasiparticle resonance disappears and a metal-insulator transition occurs. ${ }^{12}$ This approach has been successfully applied to a number of transition metal-oxides, ${ }^{49,51}$ transition metals, ${ }^{52}$ and elemental $\mathrm{Pu}$ and Ce. ${ }^{53}$ For more details and an introduction to the LDA+DMFT approach we refer the reader to Ref. 27.

In the present paper, we solve the multi-band Anderson impurity model by QMC, ${ }^{54}$ where by means of the Trotter discretization and Hubbard-Stratonovich transformations the interacting Anderson impurity model is mapped to a sum of non-interacting problems, the sum being performed by the Monte-Carlo technique. We employ a Trotter discretization of $\Delta \tau=0.25 \mathrm{eV}^{-1}$ unless noted otherwise and follow Ref. 55 for the Fourier transformation between Matsubara frequencies and imaginary time $\tau$. To obtain the physically relevant spectral function we analytically continue the Green function from Matsubara frequencies to real frequencies by means of the maximum entropy method. ${ }^{56}$ The QMC has the advantage of being numerically exact while the main disadvantage is that it is restricted to higher temperatures. The room temperature calculations of this paper were computationally very expensive, requiring about 200000 hours CPU time on the Hitachi SR8000-F1 at the Leibnitz Rechenzentrum Munich. For the implementation of QMC in the context of LDA+DMFT, including flow diagrams, see also Ref. 27.

\section{LDA+DMFT SPECTRA}

Using the crystal structure of paramagnetic metallic (PM) $\quad \mathrm{V}_{2} \mathrm{O}_{3}$ and paramagnetic insulating (PI) $\left(\mathrm{V}_{0.962} \mathrm{Cr}_{0.038}\right)_{2} \mathrm{O}_{3}$, respectively, as input, we performed LDA+DMFT(QMC) calculations with one $a_{1 g}$ and two degenerate $e_{g}^{\pi}$ bands. The results for the spectra of the $t_{2 g}$ bands are shown in Fig. 9. At $U=4.5 \mathrm{eV}$ both crystal structures lead to spectra showing metallic behavior, with a lower Hubbard band at about $-1 \mathrm{eV}$, an upper Hubbard band at $4 \mathrm{eV}$ and a quasiparticle peak at the Fermi edge $(0 \mathrm{eV})$. The peak at about $1 \mathrm{eV}$ is split from the upper $t_{2 g}$ Hubbard bands due to Hund's rule exchange as we will discuss below.

By contrast, at $U=5.5 \mathrm{eV}$, both crystal structures lead to spectra showing nearly insulating behavior. The lower Hubbard band is strongly enhanced whereas at the Fermi edge, a pseudo-gap is formed. Above the Fermi energy, only small changes of the two-peak structure are visible.

Apparently, qualitatively different spectra for the two crystal structures require an intermediate value of $U$. This is indeed observed at $U=5.0 \mathrm{eV}$ : Whereas pure $\mathrm{V}_{2} \mathrm{O}_{3}$ now shows a small peak at the Fermi edge (a residue of the quasiparticle peak obtained at $U=4.5 \mathrm{eV}$ ) and is therefore metallic, the Cr-doped system exhibits a pronounced minimum in the spectrum implying that

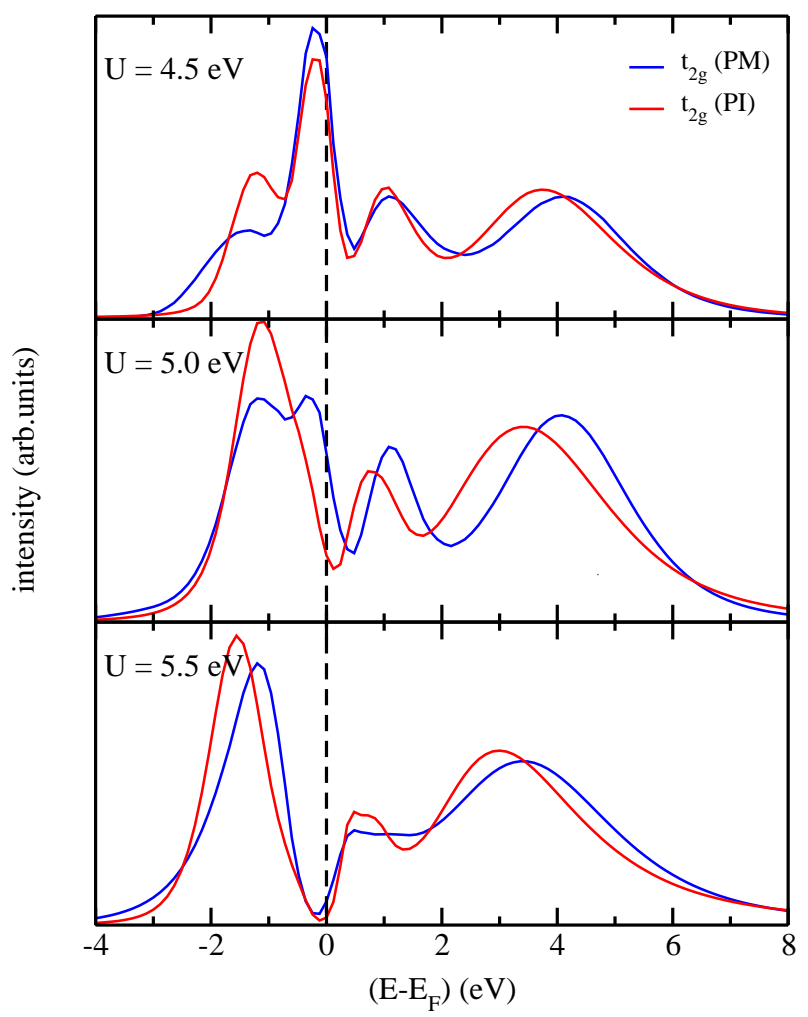

FIG. 9: LDA+DMFT(QMC) spectra for paramagnetic insulating (PI) $\left(\mathrm{V}_{0.962} \mathrm{Cr}_{0.038}\right)_{2} \mathrm{O}_{3}$ and metallic (PM) $\mathrm{V}_{2} \mathrm{O}_{3}$ at $U=4.5,5,5.5 \mathrm{eV}$, and $T=1160 \mathrm{~K}$.

it is nearly insulating. Due to the high temperature of $T=0.1 \mathrm{eV} \approx 1160 \mathrm{~K}$ of the QMC simulations one only observes a smooth crossover between the two phases with a metal-like and insulator-like behavior of the respective curves instead of a sharp metal-insulator transition as would be expected for temperatures below the critical point (i.e., for $T<400 \mathrm{~K}$ in the experiment). The value of the critical interaction of $5.0 \mathrm{eV}$ is in accordance with constrained LDA calculations by Solovyev et al. ${ }^{48}$ who analyzed the charging energy between di- and trivalent vanadium ions in an octahedral oxygen environment for $\mathrm{LaVO}_{3}$, obtaining a $U$ value for the $t_{2 g}$ orbitals which is only slightly smaller than $5 \mathrm{eV}$. Similar $U$ values of $4-5 \mathrm{eV}$ were obtained by fitting spectroscopy data for vanadium oxides to model calculations. ${ }^{57}$

Not only the overall $t_{2 g}$ DOS but also the bandresolved spectra of the $a_{1 g}$ and $e_{g}^{\pi}$ bands provide valuable insight. In Fig. 10, the $a_{1 g}$ and one of the two degenerate $e_{g}^{\pi}$ spectra are shown at $U=5.5 \mathrm{eV}$. The basic features of the spectrum can be understood as follows: We will show in the next section that the predominant local configuration has two spin-aligned electrons in the $e_{g}^{\pi}$ orbitals, i.e., a $e_{g}^{\pi} e_{g}^{\pi}$ spin-1 configuration, with some admixture of $a_{1 g} e_{g}^{\pi}$ spin-1 configurations. Since there are more $e_{g}^{\pi}$ than $a_{1 g}$ electrons, let us for a moment disregard the $a_{1 g} e_{g}^{\pi}$ configurations. The lower Hubbard band at about $-1.5 \mathrm{eV}$ indicates the removal of an $e_{g}^{\pi}$ electron from the 


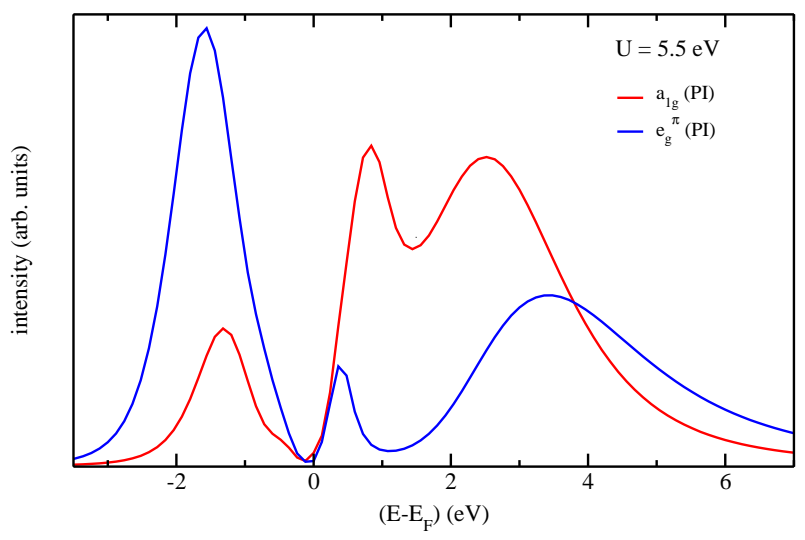

FIG. 10: LDA+DMFT(QMC) spectrum for paramagnetic insulating $\left(\mathrm{V}_{0.962} \mathrm{Cr}_{0.038}\right)_{2} \mathrm{O}_{3}(\mathrm{PI})$ for $U=5.5 \mathrm{eV} ; T=1160 \mathrm{~K}$.

predominantly $e_{g}^{\pi} e_{g}^{\pi}$ spin-1 configurations. In an atomic picture (which is a reasonable starting point for the insulating phase) this $e_{g}^{\pi} e_{g}^{\pi} \rightarrow e_{g}^{\pi}$ transition leads to an energy gain of $V-J-\mu \approx-1.5 \mathrm{eV}$ (the approximate position of the lower Hubbard band). On the other hand, the upper $e_{g}^{\pi}$ Hubbard band describes the $e_{g}^{\pi} e_{g}^{\pi} \rightarrow e_{g}^{\pi} e_{g}^{\pi} e_{g}^{\pi}$ transitions. Since the spin-alignment is lost this transition costs an energy $U+V-\mu=U+J+(V-J-\mu) \approx 4.4$ $\mathrm{eV}$, which roughly agrees with the position of the upper Hubbard band. On the other hand, adding an $a_{1 g}$ electron costs $2 V-\mu \approx 2.6 \mathrm{eV}$ or $2 V-2 J-\mu \approx 0.7 \mathrm{eV}$, depending on whether this electron is spin aligned or not. This Hund's rule splitting results in the two-peak structure of the upper $a_{1 g}$ Hubbard band in Fig. 10. Since there are also $a_{1 g} e_{g}^{\pi}$ spin-1 configurations, one has some modifications of this picture, in particular, there is also a splitting of the upper $e_{g}^{\pi}$ band, resulting in a small peak at $U \approx 0.5 \mathrm{eV}$ due to $a_{1 g} e_{g}^{\pi} \rightarrow a_{1 g} e_{g}^{\pi} e_{g}^{\pi}$ transitions. As a consequence of the splitting of the upper Hubbard band into a two-peak structure, leading to a peak at about $0.5 \mathrm{eV}$ above the Fermi edge, the gap in the insulating phase is very small, much smaller than $V \approx 3 \mathrm{eV}$ which would be expected in a one-band Hubbard model. This also explains the puzzle in the attempt to model the optical gap with a one-band Hubbard model ${ }^{18}$ : fitting to the small experimental gap one is led to an unrealistically small Coulomb repulsion of about $1 \mathrm{eV}$ and a bandwidth of less than $0.5 \mathrm{eV}$.

To study the metal-insulator transition at experimentally relevant temperatures, we performed calculations at $T=700 \mathrm{~K}$ and $T=300 \mathrm{~K}$. Since the computational effort is proportional to $T^{-3}$, those low temperature calculations were computationally very expensive. Fig. 11 shows the results of our calculations at $T=1160 \mathrm{~K}$, $T=700 \mathrm{~K}$, and $T=300 \mathrm{~K}$ for metallic $\mathrm{V}_{2} \mathrm{O}_{3}$ and for insulating $\left(\mathrm{V}_{0.962} \mathrm{Cr}_{0.038}\right)_{2} \mathrm{O}_{3}$. In the metallic phase, the incoherent features are hardly affected when the temperature is changed, whereas the quasiparticle peak becomes sharper and more pronounced at lower temperatures. This behavior also occurs in the Anderson impu- rity model $^{58}$ and has its origin in the smoothing of the Abrikosov-Suhl quasiparticle resonance at temperatures larger than the Kondo temperature. However, in contrast to the Anderson impurity model this smoothing occurs at considerably lower temperatures which is apparently an effect of the DMFT self-consistency cycle.

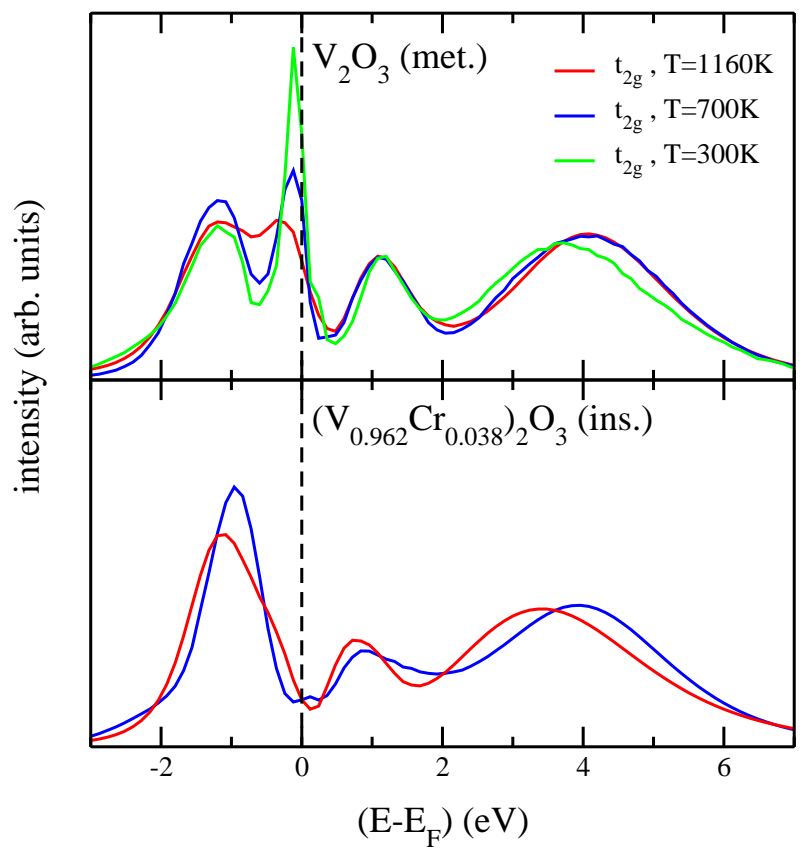

FIG. 11: LDA+DMFT(QMC) spectra for paramagnetic insulating $\left(\mathrm{V}_{0.962} \mathrm{Cr}_{0.038}\right)_{2} \mathrm{O}_{3}$ and metallic $\mathrm{V}_{2} \mathrm{O}_{3}$ at $U=5 \mathrm{eV}$.

To study the possible effect of a smaller Hund's rule coupling $J$, we performed additional calculations for a reduced value of $J=0.7 \mathrm{eV}$, keeping $V$ nearly constant. The results in Fig. 12 show that the positions of the upper Hubbard bands are significantly shifted towards lower energies while the spectra below the Fermi energy are hardly affected. This suggests that the physical properies do not change much. Indeed, we find, for example, that the spin-1 state hardly changes when the Hund's exchange is reduced. Even at values as low as $J=0.5 \mathrm{eV}$ the local moment stays almost maximal, i.e., $\left\langle m_{z}^{2}\right\rangle=3.85$ at $J=0.5 \mathrm{eV}$, implying that unrealistically small values of $J$ are required for the Castellani et al. ${ }^{36}$ picture to hold. ${ }^{59}$

\section{CHANGES ACROSS THE MOTT-HUBBARD TRANSITION}

\section{A. Local magnetic moment and orbital occupation}

The spin and orbital degrees of freedom play an important role in the paramagnetic phase of $\mathrm{V}_{2} \mathrm{O}_{3}$ and in the changes occurring across the MIT. For example, we find the squared local magnetic moment $\left\langle m_{z}^{2}\right\rangle=$ 


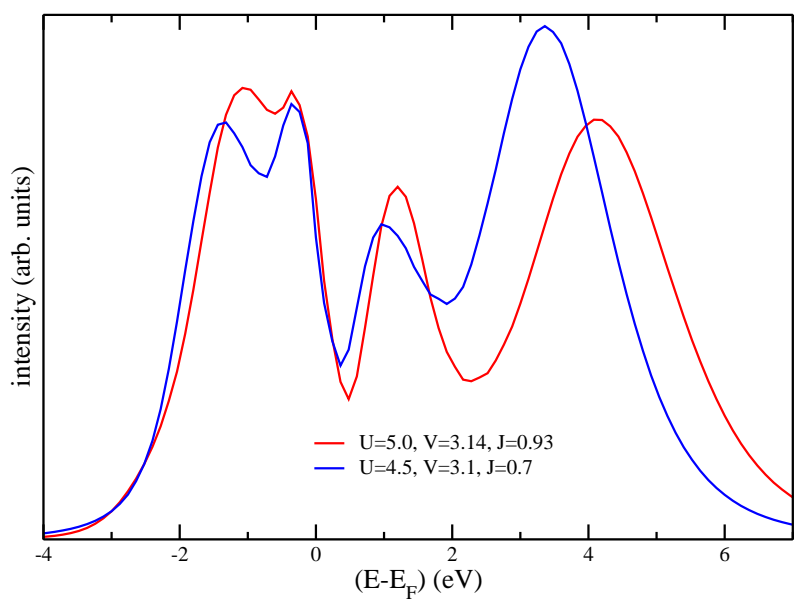

FIG. 12: Comparison of the LDA+DMFT(QMC) $\mathrm{V}_{2} \mathrm{O}_{3}$ spectra at two strengths of the exchange interaction: $J=0.93 \mathrm{eV}$ (as obtained from constrained LDA), $U=5.0 \mathrm{eV}, V=$ $3.14 \mathrm{eV}$, and $J=0.7 \mathrm{eV}, U=4.5 \mathrm{eV}, V=3.1 \mathrm{eV}$.

$\left\langle\left(\sum_{m}\left[\hat{n}_{m \uparrow}-\hat{n}_{m \downarrow}\right]\right)^{2}\right\rangle$ to have a value of $\left\langle m_{z}^{2}\right\rangle \approx 4$, unaffected by the MIT, see Fig. 13. This value corresponds to two spin-aligned electrons in the $\left(a_{1 g}, e_{g 1}^{\pi}, e_{g 2}^{\pi}\right)$ orbitals and therefore to a spin- 1 state in the Mott-Hubbard transition regime in good agreement with polarization dependent x-ray absorption measurements of Park et al. ${ }^{37} \mathrm{It}$ also agrees with measurements of the high temperature susceptibility which give the value of $\mu_{\text {eff }}=2.66 \mu_{B}$ for the effective magnetic moment. ${ }^{60}$ This is close to the ideal $S=1$ value $\mu_{e f f}=2.83 \mu_{B}$. Note that when $U$ is reduced to $U<3$, the Hund's rule coupling $J$ needs to be reduced as well to avoid an unphysical attractive Coulomb interaction (namely, a Coulomb energy $U-3 J<0$ would otherwise be gained when a spin-aligned electron is added to a singly occupied site). It is this reduction of $J$ which finally leads to a smaller local squared magnetic moment. Our results of a spin state which is essentially unaffected by the MIT is in stark contrast to results for the oneband Hubbard model where $m_{z}^{2}$ changes strongly at the MIT $^{13}$ (in fact, this quantity had even been used as an indicator for the MIT).

The orbital occupation (Fig. 13) obtained by us clearly rules out a $a_{1 g}$ singlet since this would correspond to $n_{a_{1 g}}=1, n_{e_{g}^{\pi}}=1$. Therefore our results contradict the model of Castellani et al. ${ }^{36}$ who proposed the formation of an $a_{1 g}$ singlet and hence a spin- $1 / 2$ state. At all $U$-values we find predominantly occupied $e_{g}^{\pi}$ orbitals, but with a significant admixture of $a_{1 g}$ orbitals (see Fig. 13). On the basis of an analysis of their linear dichroism data Park et al. ${ }^{37}$ concluded that the ratio of the configurations $\left(e_{g}^{\pi}, e_{g}^{\pi}\right)$ and $\left(e_{g}^{\pi}, a_{1 g}\right)$ is equal to $1: 1$ for the paramagnetic metallic phase (PM) and 3:2 for the paramagnetic insulating phase $(\mathrm{PI})$. This corresponds to an electron occupation of the $\left(a_{1 g}, e_{g 1}^{\pi}, e_{g 2}^{\pi}\right)$ orbitals of $(0.4,0.8,0.8)$ for the PI phase and $(0.5,0.75,0.75)$ for the $\mathrm{PM}$ phase. At $T=1160 \mathrm{~K}$ we find for the PI phase

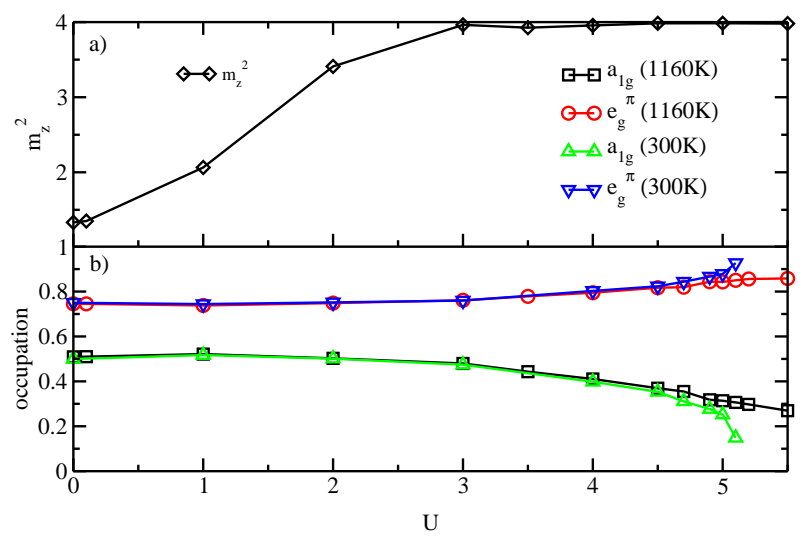

FIG. 13: a) Spin, and b) orbital occupation vs. Coulomb interaction $U$ for metallic $\mathrm{V}_{2} \mathrm{O}_{3}$.

("insulating" crystal structure and $U=5.0 \mathrm{eV}$ ) occupations of $(0.28,0.86,0.86)$, while for the metallic phase ("metallic" crystal structure and $U=5.0 \mathrm{eV}$ ) we obtain $(0.37,0.815,0.815)$. While our results give a smaller value for the admixture of $a_{1 g}$ orbitals (even more so at $T=300 \mathrm{~K}$ ), the tendency for the decrease of this value at the transition to the insulating state is well reproduced. Fig. 13 also shows that in the immediate vicinity of the Mott transition the orbital occupation has a considerable temperature dependence, with even fewer electrons in the $a_{1 g}$ orbitals at lower temperatures.

Further experimental evidence for a $\left(e_{g}^{\pi}, e_{g}^{\pi}\right)$ configuration in the ground state of the $\mathrm{V}^{+3}$ ions in $\mathrm{V}_{2} \mathrm{O}_{3}$ comes from Brown et al. ${ }^{61}$ They measured the spatial distribution of the field-induced magnetization in paramagnetic $\mathrm{V}_{2} \mathrm{O}_{3}$ by polarized neutron diffraction. Their results show that the moment induced on the $\mathrm{V}$ atoms is almost entirely due to the electrons in the doubly degenerate $e_{g}^{\pi}$ orbitals with only a minor contribution from the $a_{1 g}$ orbital. For the antiferromagnetic insulating phase of $\mathrm{V}_{2} \mathrm{O}_{3}$, calculations of the electronic structure by LDA $+\mathrm{U}$ also yielded a spin- 1 ground state for the $\mathrm{V}^{3+}$ ion and a $\left(e_{g}^{\pi}, e_{g}^{\pi}\right)$ orbital configuration. ${ }^{38}$

The origin for the ground state orbital configuration discussed above is easily understood from the LDA DOS (Fig. 5) where the center of gravity of the $a_{1 g}$ orbital is $0.3 \mathrm{eV}$ higher in energy than the corresponding value for $e_{g}^{\pi}$ orbitals. This shift together with the asymmetry of the DOS leads to an LDA occupation of about 0.55 for the $a_{1 g}$ and 0.72 for each of the $e_{g}^{\pi}$ bands. The occupation of $e_{g}^{\pi}$ orbitals is further enhanced in the strongly correlated metallic regime and, in particular, in the insulating phase where the Coulomb interaction value $(U>5 \mathrm{eV})$ is significantly larger than the bandwidth $(W \approx 2 \mathrm{eV})$. 


\section{B. Quasiparticle renormalization and spectral weight at the Fermi level}

To study the MIT in detail we have calculated the quasiparticle weight $Z$ by fitting a third order polynominal to the imaginary part of the QMC self-energy $\operatorname{Im} \Sigma\left(i \omega_{n}\right)$ at the lowest Matsubara frequencies $\omega_{n}$ which gives $Z=(1-\partial \operatorname{Im} \Sigma(i \omega) / \partial i \omega)^{-1}$ via the slope of the polynominal at $\omega=0$. The resulting quasiparticle weight for the $a_{1 g^{-}}$and the $e_{g}^{\pi}$-bands is shown as a function of $U$ in Fig. 14. With increasing $U, Z$ first shows a strong decrease for both types of bands. However, in the vicinity of the MIT at about $U=5 \mathrm{eV}$ the $a_{1 g}$ quasiparticle weight remains constant while that of the $e_{g}^{\pi}$ electrons goes to zero. This behavior of the $a_{1 g}$ quasiparticle weight is in striking contrast to the behavior at the MIT in the oneband Hubbard model where $Z \rightarrow 0$, such that its inverse, the effective mass, diverges. Indeed, from Fig. 14 alone one might conclude that the MIT occurs only for the $e_{g}^{\pi}$ band. On the other hand, the total LDA+DMFT spectrum clearly shows insulating behavior at large Coulomb interactions.

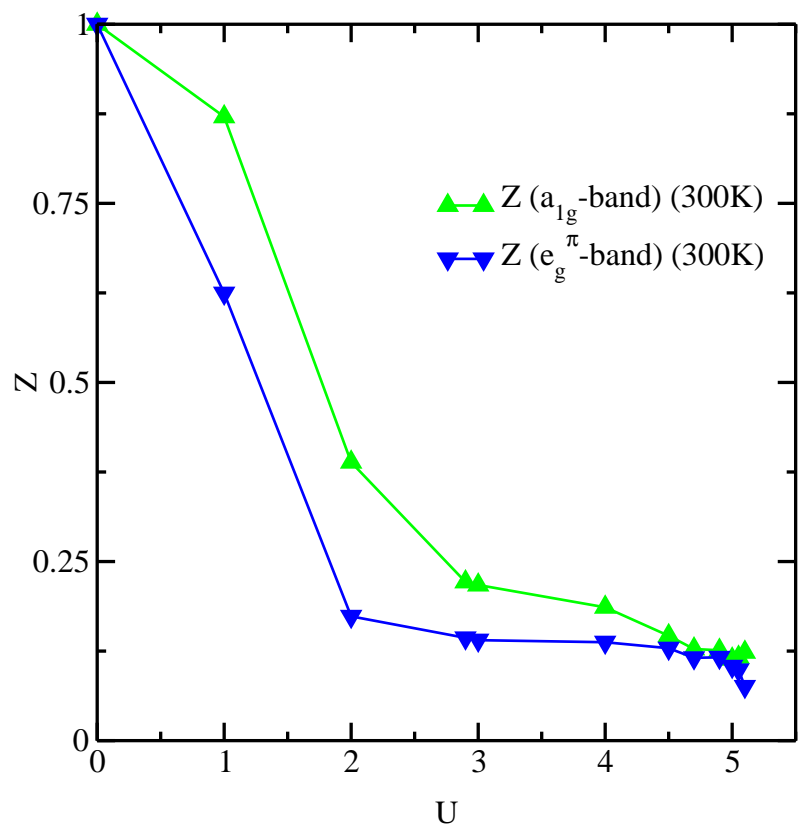

FIG. 14: Quasiparticle weight $Z$ for the $a_{1 g}$ and the $e_{g}^{\pi}$ bands vs. $U$, using the crystal structure of metallic $\mathrm{V}_{2} \mathrm{O}_{3}$.

A quantity which measures the spectral weight at the Fermi energy and does not dependent on the analytical continuation to real frequencies is given by

$$
-\frac{\beta}{\pi} G(\tau=\beta / 2)=\int A(\omega) \underbrace{\frac{\beta}{\pi} \frac{\exp (-\beta / 2 \omega)}{1+\exp (-\beta \omega)}}_{K(\omega)} \mathrm{d} \omega .
$$

More specifically, $-\beta / \pi G(\tau=\beta / 2)$ measures $A(\omega)$ in the region given by the kernel $K(\omega)$ which is centered around the Fermi energy at $\omega=0$ and has a width proportional to $T=1 / \beta$. The results in Fig. 15 show that, for 300 $\mathrm{K}$, the spectral weight at the Fermi energy disappears at a critical value of $U$ between 5.1 and $5.2 \mathrm{eV}$ for both types of orbitals in the case of metallic $\mathrm{V}_{2} \mathrm{O}_{3}$. These values of $U$ agree quite well with the position where the $e_{g}^{\pi}$ quasiparticle weight is expected to disappear in Fig. 14. For the $300 \mathrm{~K}$ data for insulating $\left(\mathrm{V}_{0.962} \mathrm{Cr}_{0.038}\right)_{2} \mathrm{O}_{3}$, the critical U-value is between 4.9 and $5.0 \mathrm{eV}$. With increasing temperature, the MIT is a smeared out to become a crossover and, at $1160 \mathrm{~K}$, is only signaled by a change of curvature slightly above $5 \mathrm{eV}$.

This analysis reaffirms the correctness of our choice of the value $U=5 \mathrm{eV}$ for the simultaneous description of the metallic and insulating behavior of $\mathrm{V}_{2} \mathrm{O}_{3}$ and $\left(\mathrm{V}_{0.962} \mathrm{Cr}_{0.038}\right)_{2} \mathrm{O}_{3}$, respectively.

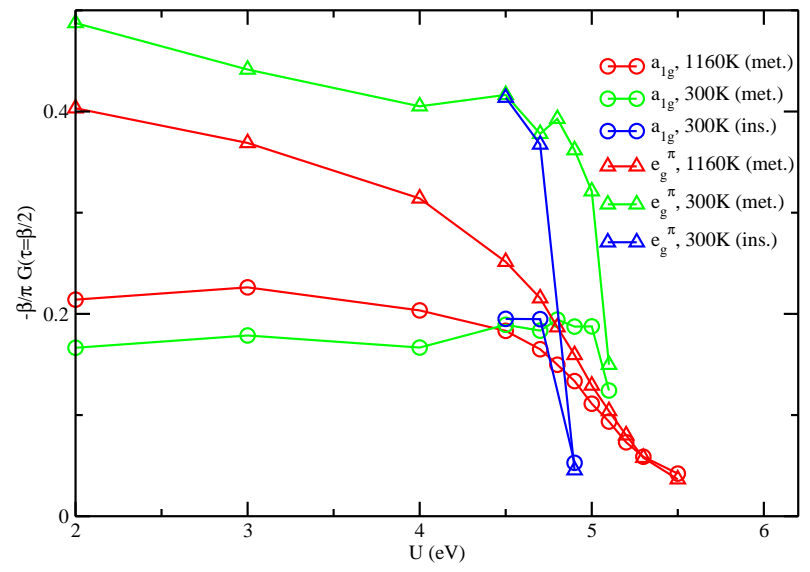

FIG. 15: Spectral weight of the $a_{1 g}$ and $e_{g}^{\pi}$ orbitals at the Fermi energy, as estimated by $-\beta / \pi G(\tau=\beta / 2)$, vs. $U$.

We still have to address the question why the $a_{1 g}$ quasiparticle weight remains constant across the transition, i.e., why the effective $a_{1 g}$ mass does not diverge. The DMFT Green function is given by Eq. (2) which under the assumption of a Fermi liquid-like self-energy

$$
\Sigma_{m}(\omega)=\operatorname{Re} \Sigma_{m}(0)+\left.\frac{\partial R e \Sigma_{m}(\omega)}{\partial \omega}\right|_{\omega=0} \omega
$$

and $Z=\left(1-\partial \operatorname{Re} \Sigma_{m}(\omega) /\left.\partial \omega\right|_{\omega=0}\right)^{-1}$ yields

$$
G_{m}(\omega)=\int \mathrm{d} \epsilon \frac{Z N_{m}^{0}(\epsilon)}{\omega+Z\left(\mu-\operatorname{Re} \Sigma_{m}(0)-\epsilon\right)} .
$$

Hence, an MIT can either occur if the effective mass diverges, i.e., $Z \rightarrow 0$, or if the effective chemical potential $\mu-\operatorname{Re} \Sigma_{m}(0)$ moves, due to electronic correlations, outside the non-interacting LDA DOS such that $N_{m}^{0}\left(\mu-\operatorname{Re} \Sigma_{m}(0)\right)=0$. In the case of $\mathrm{V}_{2} \mathrm{O}_{3}$ the latter happens as is demonstrated by Fig. 16, where $\operatorname{Re} \Sigma_{m}(0)$ has been approximated by its value at the lowest Matsubara frequency, $\operatorname{Re} \Sigma_{m}\left(\omega_{0}\right)$. At the MIT, between $U=5.1$ and $5.2 \mathrm{eV}, \mu-\operatorname{Re} \Sigma_{a_{1 g}}(0)$ crosses the upper LDA band edge while $\mu-\operatorname{Re} \Sigma_{e_{g}^{\pi}}(0)$ moves below the lower band edge. 
This explains the pronounced changes of the orbital occupation in Fig. 13 and, in particular, why an MIT can occur although the $a_{1 g}$ quasiparticle weight does not vanish (Fig. 14). This unexpected feature of the MIT has important physical consequences: Since at the MIT $N_{a_{1 g}}^{0}\left(\mu-\operatorname{Re} \Sigma_{a_{1 g}}(0)\right) \rightarrow 0$, the height of the $a_{1 g}$ quasiparticle peak goes to zero, rather than its width which is given by $Z$. For the $e_{g}^{\pi}$ band we have both $N_{e_{g}^{\pi}}^{0}\left(\mu-\operatorname{Re} \Sigma_{e_{g}^{\pi}}(0)\right) \rightarrow 0$ and $Z \rightarrow 0$ such that height and width simultaneously go to zero. Therefore the quasiparticle DOS $N_{a_{1 g}}^{0}\left(\mu-\operatorname{Re} \Sigma_{a_{1 g}}(0)\right) / Z$ does not diverge. Consequently, physical quantities proportional to this quasiparticle DOS like the linear coefficient of the specific heat and the local susceptibility do not diverge, at least for the $a_{1 g}$ bands.

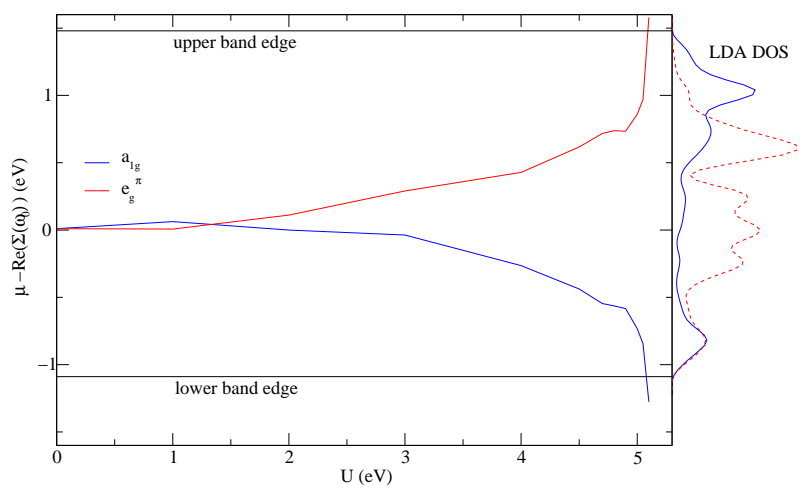

FIG. 16: Effective chemical potential $\mu-\operatorname{Re} \Sigma\left(\omega_{0}\right)$ vs. $U$. The upper and lower band edges of the non-interacting LDA DOS are shown as solid lines and the entire LDA DOS of $\mathrm{V}_{2} \mathrm{O}_{3}$ is plotted vertically at the right $\mathrm{z}$-axis.

\section{COMPARISON WITH EXPERIMENTAL SPECTRA}

To be able to compare with experimental photoemission spectra the LDA+DMFT results were multiplied with the Fermi function at the experimental temperature $(T \approx 180 \mathrm{~K})$ and broadened with a $0.09 \mathrm{eV}$ Gaussian to account for the experimental resolution. ${ }^{28}$ The same procedure was used for the comparison with x-ray spectroscopy data (with an inverse Fermi function at $T=300 \mathrm{~K}$ and a broadening of $0.2 \mathrm{eV}$ taken from experiment). On the experimental side, the PES of Refs. 28,62 were corrected for the inelastic Shirley-type background which also removes the $\mathrm{O} 2 p$ contribution. All experimental and theoretical curves were normalized to yield the same area (which is a measure of the occupation of the vanadium $t_{2 g}$ bands).

In Fig. 17, LDA+DMFT results for temperatures $1160 \mathrm{~K}, 700 \mathrm{~K}$, and $300 \mathrm{~K}$ are presented. Besides the broad, essentially temperature independent peak at about $-1.25 \mathrm{eV}$ corresponding to the lower Hubbard band, the three curves clearly show the development of a

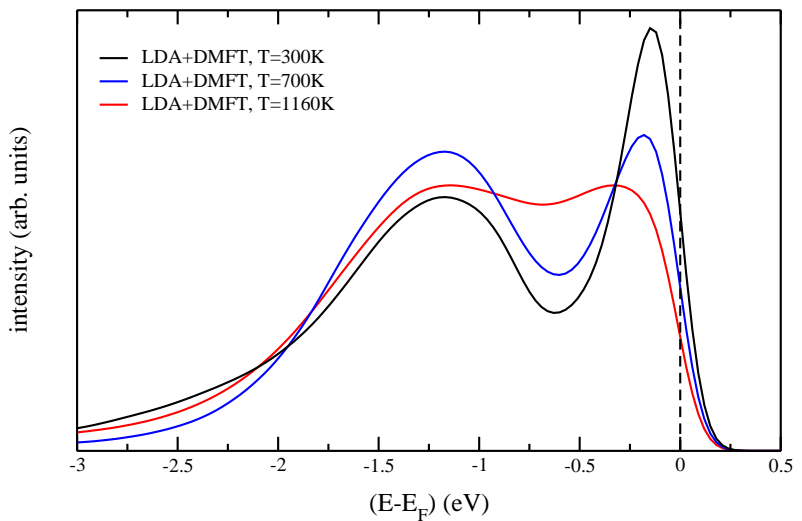

FIG. 17: LDA+DMFT(QMC) results for the metallic phase at different temperatures.

well-defined resonance-like structure just below the Fermi energy when the temperature is decreased. The latter peak is what remains of the quasiparticle peak after multiplication with the Fermi function. At $1160 \mathrm{~K}$ it is nearly equal in height to the lower Hubbard band, and there remains almost no minimum between these two features.

In Fig. 18, the LDA+DMFT results at $300 \mathrm{~K}$ are compared with early photoemission spectra by Schramme ${ }^{62}$ and recent high-resolution bulk-sensitive photoemission spectra by Mo et al. ${ }^{28}$ The strong difference between the experimental results is now known to be due to the distinct surface sensitivity of the earlier data. In fact, the photoemission data by Mo et al. ${ }^{28}$ obtained at $h \nu=700 \mathrm{eV}$ and $T=175 \mathrm{~K}$ exhibit, for the first time, a pronounced quasiparticle peak. This is in good qualitative agreement with our low temperature calculations. However, the experimental quasiparticle peak has more spectral weight. The origin for this discrepancy, for a system as close to a Mott transition as $\mathrm{V}_{2} \mathrm{O}_{3}$, is presently not clear.

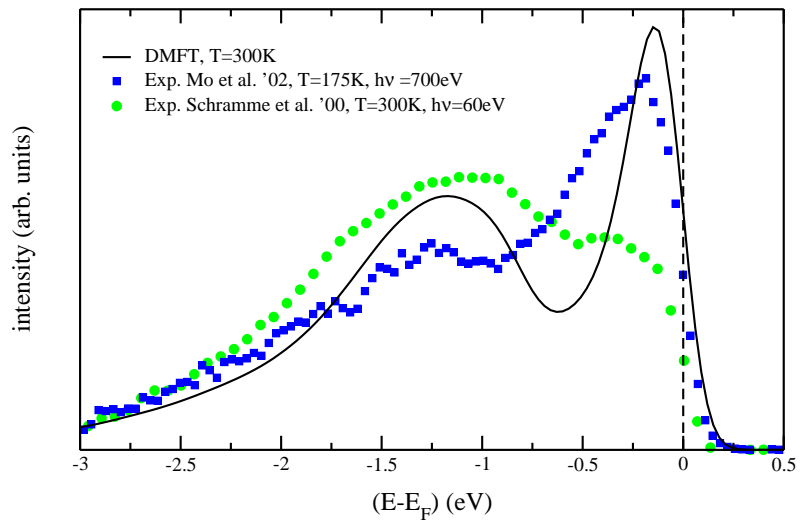

FIG. 18: Comparison of LDA+DMFT(QMC) results at $T=$ $300 \mathrm{~K}$ with photoemission data by Schramme et al. ${ }^{62}$ and Mo et $a l .{ }^{28}$ for metallic $\mathrm{V}_{2} \mathrm{O}_{3}$.

In Fig. 19 we present the corresponding calculations for Cr-doped $\mathrm{V}_{2} \mathrm{O}_{3}$ : There is a lower Hubbard band 
centered at about $-1 \mathrm{eV}$ as in the metallic phase, but a quasiparticle peak at the Fermi energy no longer exists. It is interesting to note, however, that there remains some spectral weight in the vicinity of the Fermi energy. Clearly this is not a Fermi liquid effect, but is due to highly incoherent states with a large imaginary part of the low-frequency self-energy. With decreasing tempera-

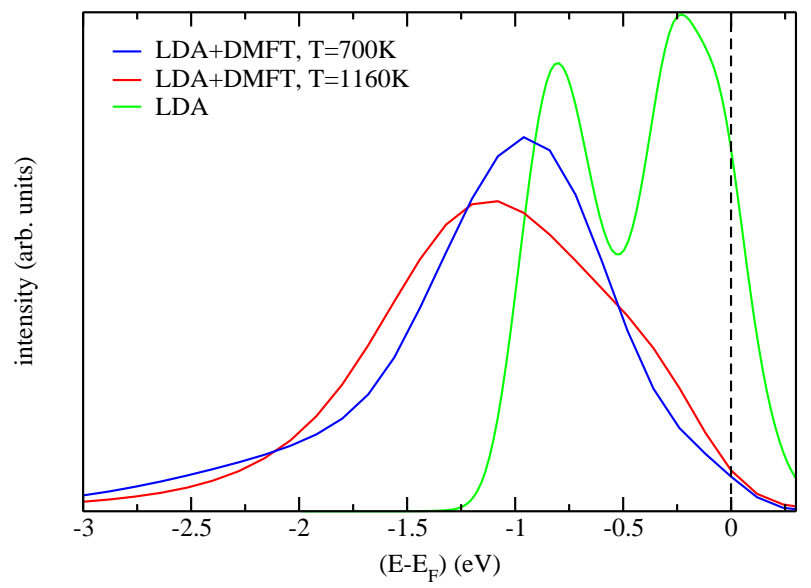

FIG. 19: Comparison of LDA and LDA+DMFT(QMC) results for insulating $\left(\mathrm{V}_{0.962} \mathrm{Cr}_{0.038}\right)_{2} \mathrm{O}_{3}$.

tures, this incoherent spectral weight is reduced and is expected to vanish for $T \rightarrow 0$. Therefore the resistance $i n$ creases with decreasing temperature as is to be expected for an insulator. For comparison we also show the LDA data in Fig. 19. They give a completely different picture: Besides a small peak at about $-0.8 \mathrm{eV}$ which is roughly in the same energy region as the lower Hubbard band of the LDA+DMFT calculations, it shows a strong peak slightly below the Fermi energy. Clearly, LDA predicts a metallic solution, although the input crystal structure is that for insulating $\left(\mathrm{V}_{0.962} \mathrm{Cr}_{0.038}\right)_{2} \mathrm{O}_{3}$.

While the comparison with PES data provides important insight into the physics of $\mathrm{V}_{2} \mathrm{O}_{3}$, more than half of the theoretical spectrum lies above $E_{F}$. For this region we compare our results at $1160 \mathrm{~K}, 700 \mathrm{~K}$, and $300 \mathrm{~K}$ with $\mathrm{O} 1 s$ X-ray absorption spectra (XAS) for $\mathrm{V}_{2} \mathrm{O}_{3}$ at $300 \mathrm{~K}$ by Müller et al. ${ }^{63}$ (see Fig. 20). Since in the XASdata the Fermi energy is not precisely determined, the data were shifted so that the peaks at $1.1 \mathrm{eV}$ coincide; all curves were normalized to the same area.

The theoretical spectra above $E_{F}$ are found to be almost independent of temperature. Just above the Fermi energy they all show some structure (i.e., a shoulder at higher temperatures developing into a small peak at low temperatures $(300 \mathrm{~K})$ ) which is the residue of the quasiparticle peak. Furthermore, at $1.1 \mathrm{eV}$ there is a rather narrow peak, and at about $4.2 \mathrm{eV}$ a broad peak. The latter two structures are parts of the upper Hubbard band which is split due to the Hund's rule coupling $J$. Hence, the relative position of those two peaks can be expected to depend sensitively on the value of $J$. A slightly smaller value of $J$ will therefore yield an even better agreement

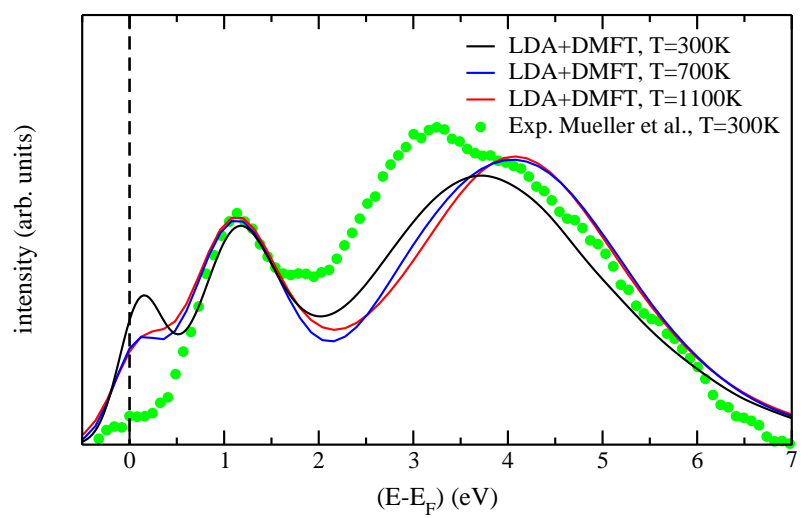

FIG. 20: Comparison of LDA+DMFT(QMC) results with Xray absorption data by Müller et al. ${ }^{63}$ for metallic $\mathrm{V}_{2} \mathrm{O}_{3}$.

with experiment.

The absence of any quasiparticle weight near $E_{F}$ in the XAS data is puzzling. This quasiparticle weight is not only present in the theoretical spectra above and below $E_{F}$, but is also seen in the high resolution PES measurements by Mo et al. ${ }^{28}$ below $E_{F}$. This calls for additional XAS or inverse photoemission spectroscopy experiments. For comparison with future experiments, we also show the theoretical XAS spectra for Cr-doped insulating $\mathrm{V}_{2} \mathrm{O}_{3}$ in Fig. 21, where our data have been broadened with the experimental resolution of Müller et al. ${ }^{63}$

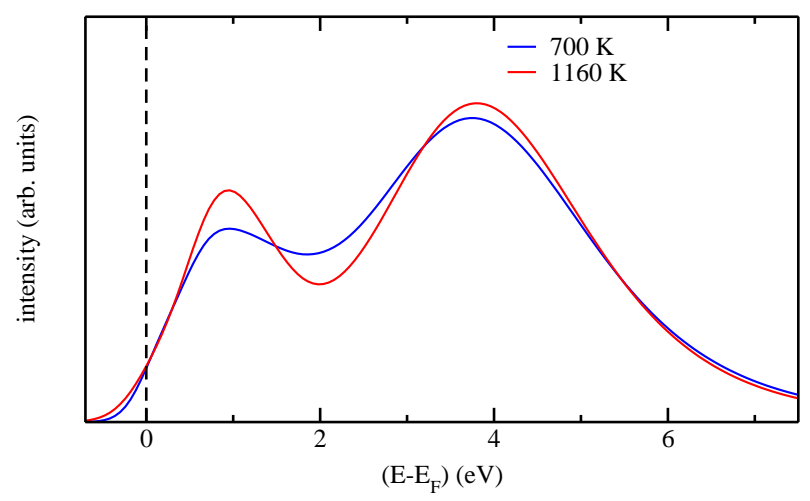

FIG. 21: LDA+DMFT(QMC) spectra for $E>E_{F}$ for insulating $\left(\mathrm{V}_{0.962} \mathrm{Cr}_{0.038}\right)_{2} \mathrm{O}_{3}$.

The comparison between theoretical and experimental spectra for metallic $\mathrm{V}_{2} \mathrm{O}_{3}$ is summed up in Fig. 22 where our LDA+DMFT results for $300 \mathrm{~K}$ are shown together with the experimental PES data by Mo et al. ${ }^{28}$ and XAS data by Müller et al. ${ }^{63}$ To document the theoretical improvement achieved by including the electronic correlations with the LDA+DMFT technique we also show the results of LDA. We note again that, by adjusting the value of $U$ such that the experimentally determined crystal structures lead to the correct metallic and insulating behavior, the spectrum was calculated without any further parameter fit. In consideration of this fact the agreement of our results with the experi- 
mental spectra above and below the Fermi energy is remarkably good. Although LDA yields the same gross

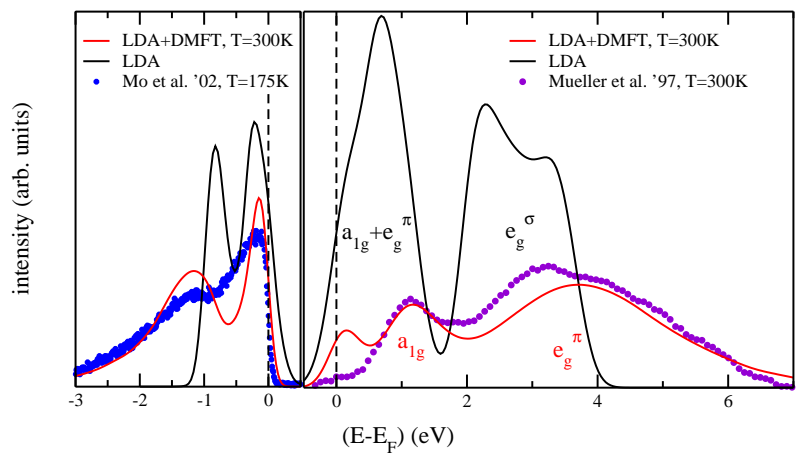

FIG. 22: Comparison of LDA+DMFT(QMC) results with PES data by Mo et al. ${ }^{28}$ and X-ray absorption data by Müller et al. ${ }^{63}$ for the metallic phase above and below $E_{F}$

features, their weight, position and width neither agree with LDA+DMFT nor experiment. The interpretation of the two large peaks in the upper half of the spectrum is also different within LDA and LDA+DMFT. As denoted in the figure, the peaks in LDA are purely from $t_{2 g}$ (lower peak) and $e_{g}^{\sigma}$ (upper peak) bands whereas they are mainly $a_{1 g}$ for the lower and $e_{g}^{\pi}$ for the upper peak in LDA+DMFT, with some admixture of the respective other band.

We note that the $e_{g}^{\sigma}$ bands were not taken into account in our calculations. Therefore, while the complete LDA curve is normalized to an area of 10 (corresponding to ten d-electrons), the experimental and LDA+DMFT curves are normalized to an area of 6 (corresponding to the six electrons of the $t_{2 g}$ bands). We may estimate the position of the $e_{g}^{\sigma}$ bands in a LDA+DMFT calculation as following: Assuming that the intra- $t_{2 g}$ Coulomb interaction $V=U-2 J \approx 3 \mathrm{eV}$ also applies for the interaction between spin-aligned $t_{2 g^{-}}$and $e_{g}^{\sigma}$-electrons, and taking the difference between the $e_{g}^{\sigma}$ - and $t_{2 g}$-band centers of gravity of roughly $2.5 \mathrm{eV}$ into account, we expect the $e_{g}^{\sigma}$-band to be located roughly at $2.5 \mathrm{eV}+3 \mathrm{eV}=5.5 \mathrm{eV}$ above the lower Hubbard band $(-1.5 \mathrm{eV})$, i.e., at about $4 \mathrm{eV}$. With this estimate we expect the (upper) XAS peak at around $4 \mathrm{eV}$ in Fig. 22 to be an admixture of $e_{g}^{\sigma}$ and $t_{2 g}$ states. More precisely, this upper Hubbard band describes transitions from $e_{g}^{\pi} e_{g}^{\pi}$ configurations with two electrons to the three-electron configurations $e_{g}^{\pi} e_{g}^{\pi} e_{g}^{\pi}, e_{g}^{\pi} e_{g}^{\pi} a_{1 g}$, and $e_{g}^{\pi} e_{g}^{\pi} e_{g}^{\sigma}$ (there is a minor admixture of $e_{g}^{\pi} a_{1 g}$ states).

The properties of paramagnetic $\mathrm{V}_{2} \mathrm{O}_{3}$ across the MIT obtained with LDA+DMFT for a multi-band model are thus found to be remarkably different from those known from the one-band Hubbard model. Indeed, the orbital degrees of freedom are seen to play an essential role: They are not only responsible for the high asymmetry of the spectra below and above the Fermi energy, but are also required to explain the reduction of the height of the quasiparticle peak at the Fermi energy when the MIT is approached in the metallic phase, as well as the smallness of the insulating gap.

\section{CONCLUSION}

Using LDA-calculated densities of states for paramagnetic metallic $\mathrm{V}_{2} \mathrm{O}_{3}$ as well as paramagnetic insulating $\left(\mathrm{V}_{0.962} \mathrm{Cr}_{0.038}\right)_{2} \mathrm{O}_{3}$ as input, we performed DMFT(QMC) calculations at $300 \mathrm{~K}, 700 \mathrm{~K}$, and $1160 \mathrm{~K}$ for various $U$ values. For $U \approx 5 \mathrm{eV}$, the calculated spectra show a Mott-Hubbard MIT (or rather a sharp crossover at the temperatures accessible by present-day QMC simulations). The details of this MIT are quite different from those obtained within the one-band Hubbard model. ${ }^{13,15-18}$ In the latter model the height of the quasiparticle peak at the Fermi energy is fixed and the MIT is signaled by a divergence of the effective mass (or the inverse quasiparticle weight $1 / Z$ ) such that the width of the quasiparticle peak goes to zero. In contrast, our LDA+DMFT results show that, for the $a_{1 g}$ quasiparticle peak, the height goes to zero while the width stays constant, as indicated by a roughly constant value of $1 / Z$ at the MIT. For the $e_{g}^{\pi}$ quasiparticle peak a combination of, both, a reduced height and width at the MIT is found. This new type of physics, but also the high asymmetry of the spectra below and above the Fermi energy as well as the smallness of the insulating gap, are all due to the orbital degrees of freedom.

We compared our theoretical data at $U=5 \mathrm{eV}$ with the results of various experimental measurements and found the orbital occupation to be predominantly of $e_{g}^{\pi}$ character (with a small admixture of $a_{1 g}$ ) in agreement with experiments. The occupation decreases for higher $U$-values, especially at low temperatures. Furthermore, we found a spin- 1 state across the MIT in agreement with polarization dependent X-ray absorption measurements. The $300 \mathrm{~K}$ spectrum calculated by us for metallic $\mathrm{V}_{2} \mathrm{O}_{3}$ is in good overall agreement with new bulk-sensitive PES measurements. ${ }^{28}$ On the other hand, the difference in the quasiparticle weight remains to be explained. The comparison with X-ray absorption measurements shows that our LDA+DMFT(QMC) calculations also give a good description of the spectrum above the Fermi energy.

All calculations described above were done using the integral over the LDA density of states (DOS) (equation (2)) to obtain the lattice Green function. For a non-cubic system, this procedure is an approximation to the exact $\mathrm{LDA}+\mathrm{DMFT}$ scheme. In the future we plan to make use of the full Hamiltonian $H^{0}$ (eq. (1)). In this way it will be possible to study the influence of correlation effects on all orbitals including the $e_{g}^{\sigma}$ orbitals and the oxygen states.

\section{ACKNOWLEDGMENTS}

We thank J. W. Allen, Th. Pruschke, M. Feldbacher, I. Nekrasov, I. S. Elfimov, A. I. Lichtenstein, 
A. I. Poteryaev and G. A. Sawatzky for valuable discussions. This work was supported in part by the Deutsche Forschungsgemeinschaft through Sonderforschungsbereich 484, and the Emmy Noether program, by the Russian
Foundation for Basic Research Grant No. RFFI-01-0217063, and by the Leibniz-Rechenzentrum, München. We thank A. Sandvik for making his maximum entropy code available to us.
1 N. F. Mott, Rev. Mod. Phys. 40, 677 (1968); MetalInsulator Transitions (Taylor \& Francis, London, 1990);

${ }^{2}$ F. Gebhard, The Mott Metal-Insulator Transition (Springer, Berlin, 1997).

3 T. M. Rice and D. B. McWhan, IBM J. Res. Develop. 14, 251 (1970).

4 D. B. McWhan and J. P. Remeika, Phys. Rev. B 2, 3734 (1970).

5 D. B. McWhan, A. Menth, J. P. Remeika, W. F. Brinkman, and T. M. Rice, Phys. Rev. B 7, 1920 (1973).

6 J. Hubbard, Proc. Roy. Soc. London A276, 238 (1963).

7 M. C. Gutzwiller, Phys. Rev. Lett. 10, 59 (1963).

8 J. Kanamori, Prog. Theor. Phys. 30, 275 (1963).

9 J. Hubbard, Proc. Roy. Soc. London A281, 401 (1964).

10 W. F. Brinkman and T. M. Rice, Phys. Rev. B 2, 4302 (1970).

11 E. H. Lieb and F. Y. Wu, Phys. Rev. Lett. 20, 1445-1448 (1968).

12 W. Metzner and D. Vollhardt, Phys. Rev. Lett. 62, 324 (1989).

13 A. Georges, G. Kotliar, W. Krauth, and M. J. Rozenberg, Rev. Mod. Phys. 68, 13 (1996).

14 W. F. Brinkman and T. M. Rice, Phys. Rev. B 2, 4302 (1970).

15 G. Moeller, Q. Si, G. Kotliar, M. J. Rozenberg, and D. S. Fisher, Phys. Rev. Lett. 74, 2082 (1995).

16 M. J. Rozenberg, R. Chitra and G. Kotliar, Phys. Rev. Lett. 83, 3498 (1999).

17 R. Bulla, Phys. Rev. Lett. 83, 136 (1999).

18 M. J. Rozenberg, G. Kotliar, H. Kajueter, G. A. Thomas, D. H. Rapkine, J. M. Honig, and P. Metcalf, Phys. Rev. Lett. 75,105 (1995).

19 M. J. Rozenberg, Phys. Rev. B 55, R4855 (1997).

20 J. E. Han, M. Jarrell, and D. L. Cox, Phys. Rev. B 58, R4199 (1998).

${ }^{21}$ K. Held and D. Vollhardt, Euro. Phys. J. B 5, 473 (1998).

${ }^{22}$ P. Limelette, A. Georges, D. Jérome, P. Wzietek, P. Metcalf, J. M. Honig, Science 302, 89 (2003).

${ }^{23}$ G. Kotliar, Eur. J. Phys. B 11, 27 (1999).

${ }^{24}$ G. Kotliar, E. Lange, M. J. Rozenberg, Phys. Rev. Lett. 84, 5180 (2000).

${ }^{25}$ K. Held, G. Keller, V. Eyert, V. I. Anisimov, D. Vollhardt, Phys. Rev. Lett. 86, 5345 (2001).

26 V. I. Anisimov, A. I. Poteryaev, M. A. Korotin, A. O. Anokhin, and G. Kotliar, J. Phys. Cond. Matter 9, 7359 (1997); A. I. Lichtenstein and M. I. Katsnelson, Phys. Rev. B 57, 6884 (1998).

27 K. Held, I. A. Nekrasov, G. Keller, V. Eyert, N. Blümer, A.K. McMahan, R.T. Scalettar, Th. Pruschke, V.I. Anisimov und D. Vollhardt, "Realistic investigations of correlated electron systems with LDA+DMFT", Psi-k Newsletter \#56, 65 (2003) [http://psi-k.dl.ac.uk/newsletters/News_56/Highlight_56.pdf] .

28 S.-K. Mo, J. D. Denlinger, H.-D. Kim, J.-H. Park, J. W. Allen, A. Sekiyama, A. Yamasaki, K. Kadono, S. Suga, Y.
Saitoh, T. Muro, P. Metcalf, G. Keller, K. Held, V. Eyert, V. I. Anisimov, D. Vollhardt, Phys. Rev. Lett. 90, 186403 (2003).

29 P. D. Dernier, J. Phys. Chem. Solids 31, 2569 (1970).

30 L. F. Mattheiss, J. Phys.: Cond. Matt. 6, 6477 (1994).

31 P. Hohenberg and W. Kohn, Phys. Rev. 136, B864 (1964).

32 W. Kohn and L. J. Sham, Phys. Rev. 140, A1133 (1965).

33 A. R. Williams, J. Kübler, and C. D. Gelatt, Jr., Phys. Rev. B 19, 6094 (1979).

${ }^{34}$ V. Eyert, Int. J. Quantum Chem. 77, 1007 (2000).

35 J. W. Allen, Phys. Rev. Lett. 36, 1249 (1976).

36 C. Castellani, C. R. Natoli, and J. Ranninger, Phys. Rev. B 18, 4945 (1978), ibid. 18, 4967 (1978), ibid. 18, 5001 (1978).

37 J.-H. Park et al., Phys. Rev. B 61, 11506 (2000).

38 S. Yu. Ezhov, V. I. Anisimov, D. I. Khomskii, and G. A. Sawatzky, Phys. Rev. Lett. 83, 4136 (1999).

${ }^{39}$ F. Mila, R. Shiina, F.-C. Zhang, A. Joshi, M. Ma, V. Anisimov, T. M. Rice, Phys Rev. Lett. 85, 1714 (2000).

40 A. Tanaka, J. Phys. Soc. Japan, 71, 1091, 2002.

41 S. Di Matteo, N. B. Perkins, and C. R. Natoli, Phys. Rev. B 65, 054413 (2002).

42 I. S. Elfimov, T. Saha-Dasgupta, M. A. Korotin, condmat/0303404

43 L. Hedin and B. Lundqvist, J. Phys. C: Solid State Phys. 4, 2064 (1971); U. von Barth and L. Hedin, J. Phys. C: Solid State Phys. 5, 1629 (1972).

44 D. M. Ceperley and B. J. Alder, Phys. Rev. Lett. 45, 566 (1980).

45 R. Bulla, T. A. Costi, D. Vollhardt, Phys. Rev. B 64, 045103 (2001).

46 V. I. Anisimov, J. Zaanen, and O. K. Andersen, Phys. Rev. B 44, 943 (1991); V. I. Anisimov, F. Aryasetiawan, and A. I. Lichtenstein, J. Phys. Cond. Matter 9, 767 (1997).

47 M. Zölfl, Ph.D. thesis, Universität Regensburg, 2001.

48 I. Solovyev, N. Hamada, K. Terakura, Phys. Rev. B 53, 7158 (1996).

49 I. A. Nekrasov, K. Held, N. Blümer, V. I. Anisimov, and D. Vollhardt, Euro. Phys. J. B 18, 55 (2000).

50 For the $t_{2 g}$ partial DOS's we disregarded the small $t_{2 g}$ contributions to the oxygen bands (in the range -9 to $-4 \mathrm{eV}$ ) and renormalized the DOS's to unity. This resembles most closely the DOS's which one would obtain if one downfolded the LDA bandstructure to an effective three-band Hamiltonian at the Fermi energy.

51 See, e.g., A. Liebsch and A. Lichtenstein, Phys. Rev. Lett. 84, 1591 (2000); I. A. Nekrasov, Z. V. Pchelkina, G. Keller, Th. Pruschke, K. Held, A. Krimmel, D. Vollhardt, V. I. Anisimov, Phys. Rev. B 67, 085111 (2003); L. Craco, M. S. Laad, and E. Müller-Hartmann, Phys. Rev. Lett. 91 156402 (2003); A. Sekiyama, H. Fujiwara, S. Imada, S. Suga, H. Eisaki, S. I. Uchida, K. Takegahara, H. Harima, Y. Saitoh, I. A. Nekrasov, G. Keller, D. E. Kondakov, A. V. Kozhevnikov, Th. Pruschke, K. Held, D. Vollhardt, and V. I. Anishimov, cond-mat/0312429; E. Pavarini, S. Bier- 
mann, A. Poteryaev, A. I. Lichtenstein, A. Georges, O. K. Andersen, and cond-mat/0309102.

52 See, e.g., A. I. Lichtenstein, M. I. Katsnelson, and G. Kotliar 87, 67205 (2001); S. Biermann, A. Dallmeyer, C. Carbone, W. Eberhardt, C. Pampuch, O. Rader, M. I. Katsnelson, and A. I. Lichtenstein, cond-mat/0112430.

53 See, e.g., S. Y. Savrasov, G. Kotliar, and E. Abrahams, Nature 410, 793 (2001); M. B. Zölfl, I. A. Nekrasov, Th. Pruschke, V. I. Anisimov, and J. Keller, Phys. Rev. Lett. 87, 276403 (2001); K. Held, A. K. McMahan, and R. T. Scalettar Phys. Rev. Lett. 87, 276404 (2001); A. K. McMahan, K. Held, and R. T. Scalettar Phys. Rev. B 67, 075108 (2003).

54 J. E. Hirsch and R. M. Fye, Phys. Rev. Lett. 56, 2521 (1986); M. Jarrell, Phys. Rev. Lett. 69, 168 (1992); M. Rozenberg, X. Y. Zhang, and G. Kotliar, Phys. Rev. Lett. 69, 1236 (1992); A. Georges and W. Krauth, Phys. Rev. Lett. 69, 1240 (1992); M. Jarrell, in Numerical Methods for Lattice Quantum Many-Body Problems, edited by D. Scalapino (Addison Wesley, New-York, 1997).

55 M. Ulmke, V. Janiš, and D. Vollhardt, Phys. Rev. B 51, 10411 (1995).

${ }^{56}$ M. Jarrell and J. E. Gubernatis, Physics Reports 269,
133 (1996).

57 A. T. Mizokawa, A. Fujimori, Phys. Rev. B 48, 14150 (1993); J. Zaanen, G. A. Sawatzky, J. Solid State Chem. $\mathbf{8 8 ,} 8$ (1990).

58 A. C. Hewson The Kondo Problem to Heavy Fermions (Cambridge University Press, Cambridge, 1993).

59 For us to recover the picture of Castellani et al. ${ }^{36}$ not only an unrealistically small value of $J$ would be required, but the LDA result would also have to be different. Namely, a splitting of the $a_{1 g}$ orbital into a binding and antibinding peak of equal weight would be necessary to obtain an $a_{1 g}$ singlet with occupation $n_{a_{1 g}}=1$, leaving an unpaired spin $s=1 / 2$ in the $e_{g}^{\pi}$ orbitals.

60 D. J. Arnold, R. W. Mires, J. Chem. Phys. 48, 2231 (1968)

61 P. J. Brown, M. M. R. Costa, K. R. A. Ziebeck, J. Phys.: Cond. Matter 10, 9581 (1998).

62 M. Schramme, Ph.D. thesis, Universität Augsburg, 2000; M. Schramme et al. (unpublished).

63 O. Müller, J.-P. Urbach, E. Goering, T. Weber, R. Barth, H. Schuler, M. Klemm, and S. Horn, Phys. Rev. B 56, 15056 (1997). 